ABSTRACT

Title of Dissertation:

THE EFFECTS OF ANCHOR-BASED SEMANTIC PRIMING ON JUDGMENTAL ANCHORING

David Paul Sleeth-Keppler, Ph.D., 2004

Dissertation Directed By:

Professor Arie W. Kruglanski, Department of Psychology

This dissertation proposes a unified conceptualization of a set of seemingly distinct judgmental anchoring effects (Tversky \& Kahneman, 1974), including numeric effects (Wilson, Houston, Etling, \& Brekke, 1996), semantic effects (e.g., Strack \& Mussweiler, 1997) as well as implausible anchoring effects (e.g., Mussweiler \& Strack, 2001a). These effects are viewed as special cases of a general, anchor-based, semantic priming mechanism. Specifically, during comparisons between the judgmental target and the anchor, judges are hypothesized to be primed with general semantic notions directly related to the anchor value. These anchorbased semantics, in turn, are hypothesized to mediate anchoring effects. Study 1 supported the hypothesis that anchor-based semantics may be responsible for producing anchoring effects by showing that considerations of implausible (vs. plausible) anchors are associated with higher levels of lexical activation of anchorrelated words (measured in a lexical decision task). This finding maps on to a larger anchoring effect obtained in the implausible vs. plausible anchoring condition. In study 2, participants were primed directly with anchor related words, resulting in a 
'mimicked' anchoring effect. Study 3 showed that associatively activated concepts, primed via a conditional rule, produce anchoring effects when the target and dimension of the judgment change between the comparative and absolute questions. Study 4 failed to support the prediction that a target change between a comparative task and related absolute numerical estimate, involving the same content domain, inhibits anchoring. Study 5 varied the typical anchoring paradigm by holding the numerical anchor constant and varying the target of the comparative question, producing a purely semantic anchoring effect. Finally, study 6 replicated and extended the findings obtained in study 5 , providing additional evidence (from a lexical decision task) that anchor-based semantic notions produce anchoring effects. General discussion focuses on distinctions between the present theory and the existing models of anchoring within the social judgment literature. 


\title{
THE EFFECTS OF ANCHOR-BASED SEMANTIC PRIMING ON JUDGMENTAL ANCHORING.
}

\author{
By
}

\author{
David Paul Sleeth-Keppler
}
Dissertation submitted to the Faculty of the Graduate School of the University of Maryland, College Park, in partial fulfillment of the requirements for the degree of
Doctor of Philosophy 2004

Advisory Committee:

Professor Arie Kruglanski, Chair

Professor Charles Stangor

Professor Harold Sigall

Assistant Professor Michael Dougherty

Professor Seppo Iso-Ahola 
(C) Copyright by

David Paul Sleeth-Keppler

2004 


\section{Dedication}

This work is dedicated to my loving and patient wife Esther and to my adorable son Damien. 


\section{Acknowledgements}

I would like to thank my advisor Arie Kruglanski for his guidance and support throughout my graduate career. As a result of his mentorship I have become a better thinker and learned to appreciate the importance of theory in advancing social psychology. I would also like to thank Hans-Peter Erb and the other members of my dissertation committee, Hal Sigall, Chuck Stangor, Mike Dougherty, and Seppo IsoAhola, for their constructive and supportive feedback. Finally, I would like to thank my parents without whom I wouldn't be here today. 


\section{List of Tables and Figures}

Table 1: $\quad$ Letter strings used in the lexical decision task (Studies 39 $1 \& 6)$.

Table 2: $\quad$ Absolute estimates of Gandhi's age by anchor and

42 plausibility (Study 1).

Table 3: $\quad$ Response latencies for lexical decisions for old and young words by anchor and plausibility (Studyl).

Table 4: $\quad$ Response latencies for lexical decisions for old and young words by anchor (Study6).

Figure 1: $\quad$ Absolute estimates by priming condition (Study 2).

Figure 2: $\quad$ Absolute estimates by rule prime and anchor (Study 3). 54

Figure 3: $\quad$ Absolute estimates of geographic knowledge (countries 61 + capitals, and US Rivers combined) by anchor, target and load (Study 4). 


\section{Table of Contents}

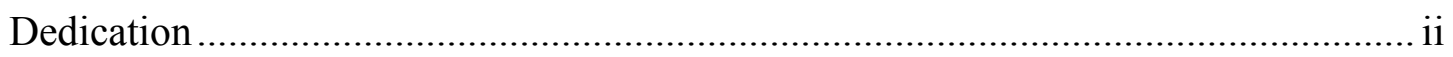

Acknowledgements..............................................................................................

List of Tables and Figures................................................................................ iv

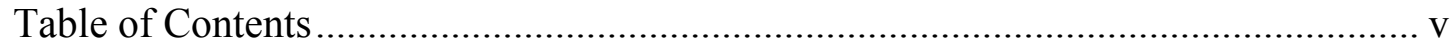

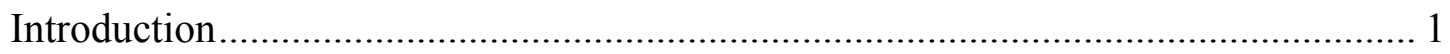

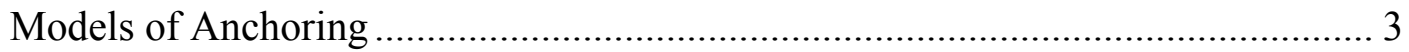

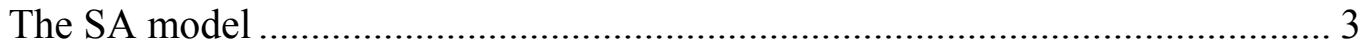

Direct measures of knowledge activation ............................................................ 6

Effects of implausible anchors.................................................................... 7

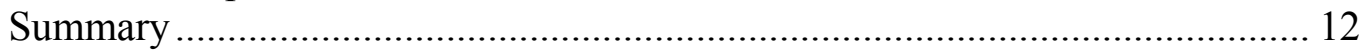

Basic anchoring as an alternative to selective accessibility............................... 12

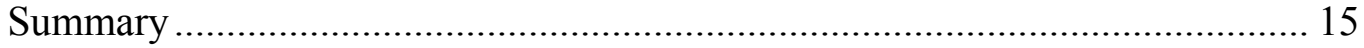

Anchor-based semantic priming: An integrative alternative .................................. 17

The semantics of anchoring revisited ........................................................... 18

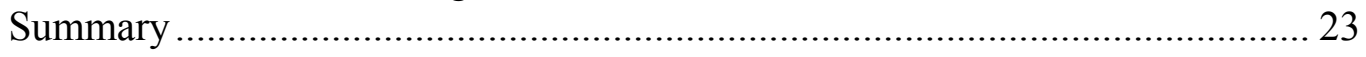

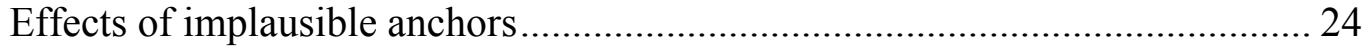

Generality of the anchoring effect ………………………………………........ 27

Rule-based linkages among concepts ........................................................... 29

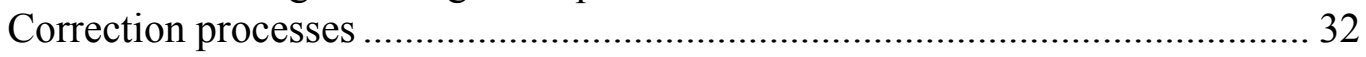

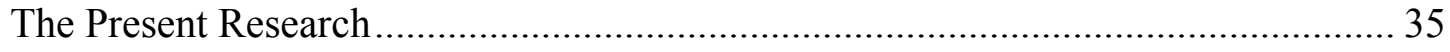

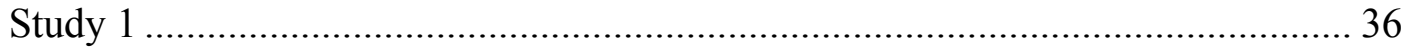

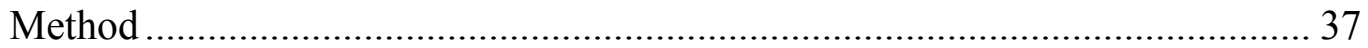

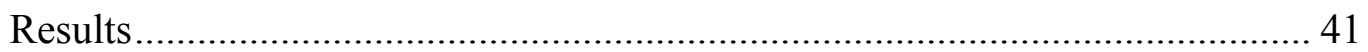

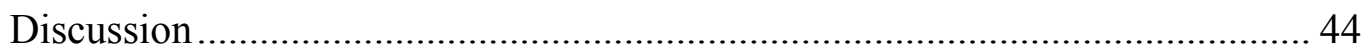

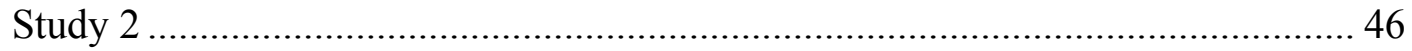

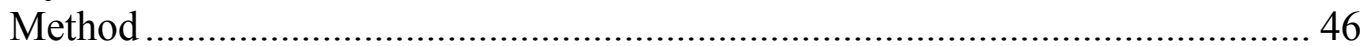

Results and Discussion .................................................................................. 48

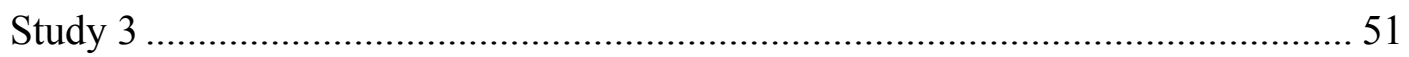

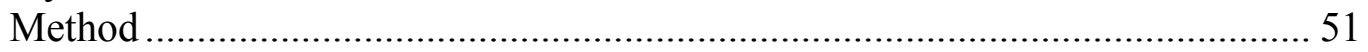

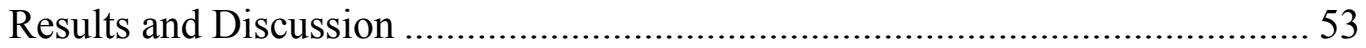

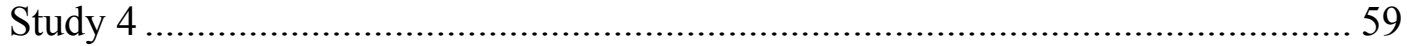

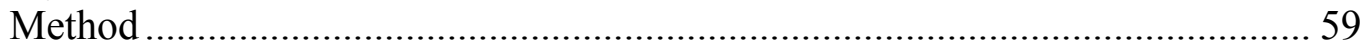

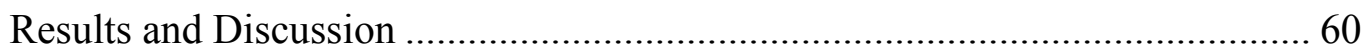

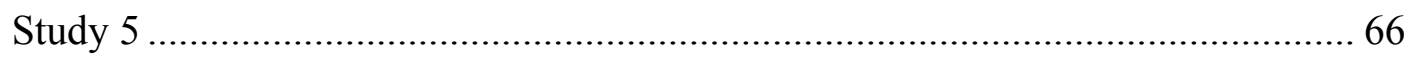

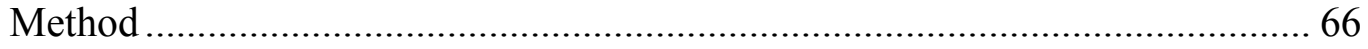

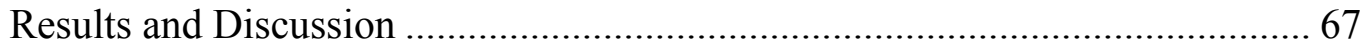




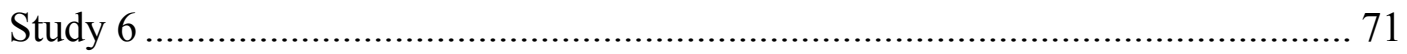

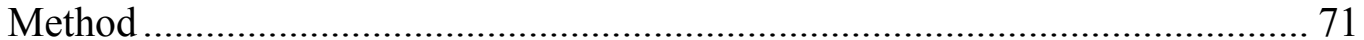

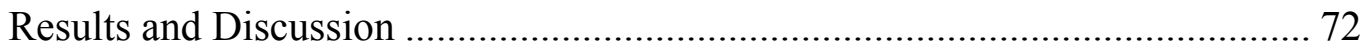

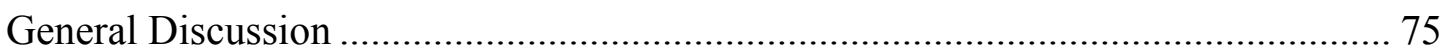

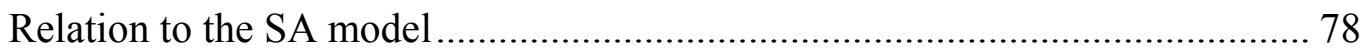

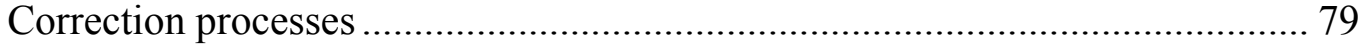

Relation to basic anchoring.................................................................... 81

Future research directions ................................................................... 84

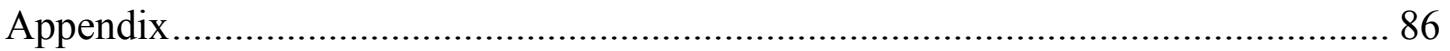

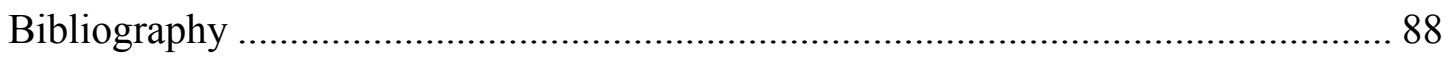




\section{Introduction}

When making judgments under conditions of uncertainty, including (but not limited to) estimations of risk, value, personal ability, and general knowledge, persons have been shown to be robustly influenced by comparison standards, or anchors. While the term anchoring is a general term that appears in a variety of classic research domains (e.g., attitude-change, see Sherif \& Hovland, 1961), social psychologists most likely associate it with the work of Tversky and Kahneman (1974), who presented insufficient adjustment and anchoring as one of three judgmental heuristics in their seminal Science article. In one of the most well known of their studies participants were first presented with a comparative question, which asked them to indicate whether the percentage of African Nations in the United Nations was higher or lower than 65 percent or 10 percent, depending on condition. Afterwards, participants were asked to provide an absolute estimate of the percentage of African Nations in the UN. Despite the fact that the anchors were generated randomly (by ways of spinning a wheel of fortune) results revealed that median estimates of the absolute percentage, $45 \%$ and $25 \%$ respectively, clearly depended on those values.

Within the field of social judgment, the anchoring and adjustment heuristic has been demonstrated in diverse areas such as self-judgments (e.g., Cervone \& Peake, 1986), negotiations (Galinsky \& Mussweiler, 2001), judicial decision-making (Chapman \& Bornstein, 1996) and in estimations of a variety of risks (e.g., Plous, 
1989), to name just a few. Furthermore, insufficient adjustment and anchoring has been used, at least analogously, to shed light on a variety of classical social psychological phenomena, including the hindsight bias (Pohl \& Hell, 1996) and the correspondence bias (Quattrone, 1982).

Initially, the main explanation for the effect came from Tversky and Kahneman (1974) themselves, who argued that judges consider the anchor value as an initial starting point for an insufficient adjustment process. That is, judges may engage in a directional adjustment process away from the anchor value and arrive (somehow) at an insufficiently adjusted absolute estimate. Jacowitz and Kahneman (1995) later elaborated on this notion and argued that the anchoring effect occurs because the directional adjustment process terminates at the nearest upper or lower boundary of a range of values a judge finds acceptable, which, from a normative standpoint, insufficiently corrects for the influence of the anchor value.

In addition to 'insufficient adjustment', a number of theories explaining the anchoring phenomenon have recently been introduced to the social psychological literature. One of the most popular of these is the selective accessibility model (SA model hereafter) of Strack and Mussweiler (1997; Mussweiler \& Strack, 1999a; 1999b; 2000a; 2000b; 2001a; 2001b; Mussweiler, Strack, \& Pfeiffer, 2000; Englich \& Mussweiler, 2001; Mussweiler, 2001; 2002; 2003). Another is Wilson, Houston, Etling, and Brekke's (1996) basic anchoring model (see also Wong \& Kwong, 2000; Brewer \& Chapman, 2002). These models paint very different pictures of the anchoring phenomenon and recently, Mussweiler and Strack (2001b) have argued that they account for different types of anchoring effects. According to these authors, 
the SA model explains primarily semantic effects, which are obtained when judgmental targets (and dimensions) stay constant between the comparative and absolute questions. Basic anchoring primarily applies to the explanation of numeric anchoring effects, which may be obtained when judgmental targets change between the comparative and absolute questions, or, in a limited number of cases, when no explicit comparison between a target and an anchor was made at all (Wilson et al., 1996; see also Brewer \& Chapman, 2002). In the present work, I seek to provide an integrative framework for these seemingly diverse effects while also providing a framework for the integration of effects of anchors that are clearly implausible, within the context of a given judgment (e.g., Mussweiler \& Strack, 2001a). Given the relative abundance of recent research and theorizing on anchoring in social psychology, it is well to review the conceptual and empirical highlights of the current major models of anchoring. Following this review I outline a new, anchor-based semantic priming perspective of judgmental anchoring effects as an integrative alternative to the currently existing models. Finally, I present six studies that test this conceptualization.

\section{Models of Anchoring}

\section{The SA model}

The SA model, which explains the anchoring effect in terms of a selective knowledge activation mechanism, assumes that when judges compare a given target to an anchor value, they may positively test the hypothesis that the target in question is identical to the anchor value. For example, when considering whether Mother 
Theresa was older or younger than 89 years at the time or her death, judges may positively test the hypothesis that she was relatively old (e.g., 89 years of age) and actively search for specific knowledge that supports this contention (e.g., she showed wrinkles on her face, walked slowly, and was less available to the media before her death). Similarly, when considering a lower value for her age (e.g., 45 years of age) judges may test the opposite hypothesis (namely that she was relatively young when she died), consequently constructing a 'younger' mental model of Mother Theresa. Thus, according to the SA model, in providing an answer to the comparative question, judges are assumed to engage in an anchor-confirmatory search of available target knowledge, which results in the activation of evidence in support of a unidirectional hypothesis (either that Mother Theresa was old in the high anchor condition or that she was young in the low anchor condition, e.g., Klayman \& Ha, 1987; Chapman \& Johnson, 1999; Strack \& Mussweiler, 1997). In the example above, estimates of Mother Theresa's age would show the typical anchoring effect as a result of the activation of different contents of target-specific knowledge (young versus old features of the judgmental target, Mother Theresa).

One of the implications of this model involves the degree to which the target knowledge, activated in response to the positive hypothesis testing process described above, is deemed relevant to a subsequent absolute estimation task (see also Higgins, 1996; Wegener \& Petty, 1995; 1997; Martin \& Achee, 1992). The SA model posits that activated target knowledge has to be applicable to a subsequent absolute judgment and representative of the target of the absolute judgment (Strack \& Mussweiler, 1997). That is, because the activated knowledge is hypothesized to be of 
considerable specificity (after all, judges presumably construct a mental model of a specific target, along a specific judgmental dimension), changes in judgmental contents between the comparative and absolute questions, involving either the dimension or the target of the judgment, should eliminate the anchoring effect, or produce absolute estimates not predicted within a standard anchoring paradigm (e.g., contrast effects).

A number of studies appear to support this selective accessibility mechanism, which has recently been extended as a general framework to explain assimilation and contrast effects in the area of comparative social judgments (Mussweiler, 2003). For example, in one study Strack and Mussweiler (1997, study 1) presented participants with either a high or a low anchor of the height of the Brandenburg Gate in Berlin (Germany). Subsequently, in a standard anchoring condition, half of the participants received an absolute estimation task involving the gate's height. For the other half of the participants, the judgmental dimension of the absolute estimation pertained to the gate's width, representing a change in dimensions between the comparative and absolute judgments. Consistent with the notion that activation of specific target knowledge mediates anchoring, an anchoring effect was obtained in the standard anchoring condition, whereas no anchoring effect was obtained in the condition in which the dimension changed between the comparative and absolute judgments, supporting the knowledge applicability constraint of the model in producing anchoring effects (Strack \& Mussweiler, 1997). In a second study, participants compared the average annual temperature in Antarctica to either a high (-20 degrees Celsius) or a low (-50 degrees Celsius) anchor. Then, depending on condition, 
participants were asked to provide an absolute estimate of either the average annual temperature in Antarctica (same target condition) or to estimate the annual average temperature in Hawaii (changed target condition). Results showed a standard anchoring effect (in the predicted direction) in the unchanged target condition, but revealed a contrast effect under conditions of changed targets. Specifically, participants in the low anchor condition gave higher temperature estimates in the Hawaii target condition, compared to participants assigned to the high anchor condition. Thus, it appears that participants may have corrected for the inherent bias of the knowledge generated as a result of the positive hypothesis test by contrasting their estimates of the average annual temperature in Hawaii away from the knowledge that became accessible to them during the comparative question, involving the judgmental target Antarctica ${ }^{1}$.

\section{Direct measures of knowledge activation}

The knowledge activation portion of the SA model is more directly supported by studies showing a reaction time advantage to anchor-consistent words in a lexical decision task. For example, in one study (Mussweiler \& Strack, 2000a, study 1) a group of German participants was presented either with a high or a low anchor of the average annual temperature in Germany, before estimating the actual mean temperature in Germany. Afterwards, all participants were exposed to a computerized

\footnotetext{
${ }^{1}$ An alternative explanation of this finding was recently put forth by Wong and Kwong (2000) who argued that participants in this study may have constructed a temporary short-term memory representation of the absolute numerical values chosen as the temperature anchors (20 and 50). Thus, the contrast effect obtained in the changed target condition may have been due to a simple numeric priming mechanism, such as the one proposed by Wilson et al. (1996), to be described in detail later, rather than the result of a judgmental correction process involving considerations of representativeness of activated target knowledge. It should be noted that this simple numeric priming mechanism only explains the contrast effect in the changed target condition, and not the anchor effect in the unchanged condition, which was in the direction predicted by the anchor values, including the prefix.
} 
lexical decision task, which contained, as part of the stimulus set, target words consistent with both the high temperature anchor (e.g., sun, hot, warm, beach) and the low temperature anchor (e.g., stove, cold, snow, frost, ski). The results showed that participants were faster at recognizing the 'summer' words in the high anchor condition (compared to participants in the low anchor condition) and faster at recognizing the 'winter' words in the low anchor condition (relative to participants in the high anchor condition). Similar results were obtained in a study varying comparison standards for the average price of a car (Mussweiler \& Strack, 2000a, study 2). Here, participants in the high versus low anchor condition showed anchorconsistent lexical activations, reflecting faster responses to expensive car brands in the high anchor condition (compared to inexpensive car brands) and faster responses to inexpensive car brands in the low anchor condition (relative to the expensive car brands). In sum, these studies demonstrate that inducing judges to consider differentially valued anchors within the context of a comparative judgment bolsters the accessibility standard consistent knowledge, which may subsequently produce anchoring effects.

\section{Effects of implausible anchors}

The SA model was initially proposed to explain primarily the effects of plausible anchor values on judgmental anchoring (see Strack \& Mussweiler, 1997). The plausibility of an anchor value is generally defined by the category to which the target of the judgment belongs. For example, in estimating whether a person is older or younger than a given anchor value, any value falling within an acceptable range of values for human age would be considered plausible. More specifically, judgments of 
plausibility can vary according to the range of values, judges would find subjectively acceptable with respect to a given judgmental category. For example, a person might consider 107 years to be a plausible value for the age of a human being, while another person might classify this value as implausible, perhaps because the person cannot think of a human that is 107 years old (see also Tversky \& Kahneman's, 1974 discussion of the availability heuristic). Similarly, the more knowledge a judge possesses about a given target, the smaller his/her subjective range of plausible values would be (Mussweiler \& Strack, 2000b). For example, if a person knew that targets belonging to the category "United States Senators" are typically between 40-60 years old, a value of 16 years would be considered implausible, despite the fact that this value would fall within the range of plausible values for human age in general. Thus, in defining whether an anchor value is plausible or implausible, it is generally important to specify which primary target category judges are likely to invoke during the judgmental task ${ }^{2}$.

An initial puzzle in the anchoring literature concerned the fact that judges have been shown to be strongly influenced by implausible anchor values in their absolute estimations of a given target feature. In one study (Strack \& Mussweiler, 1997), participants were asked whether Mahatma Gandhi was older or younger than either 140 years or 9 years of age. Despite the implausibility of these values for Gandhi's age, they produced a strong assimilation effect on subsequent absolute estimates: participants who received the high implausible anchor on average

\footnotetext{
2 The effects of differential target categorization processes on judgmental anchoring have recently been formally demonstrated by Mussweiler and Strack (2000b).
} 
estimated Gandhi to have lived 67 years, whereas participants who received the low implausible anchor thought that he was, on average, 50 years old.

Recently, Mussweiler and Strack (e.g., 2001a) have acknowledged that the SA model, in its initially proposed form, was not able to account for the effects of implausible anchors. For example, a judge can easily provide an answer to the question whether Gandhi was older or younger than 140 when he died, without first testing the hypothesis that Gandhi was 140 years old. Simply knowing that no (known) human being reaches this age suffices to answer the comparative question (at least in the high anchor condition). As a consequence, subsequent to any target comparison with an implausible anchor value, judges ought to lack the knowledge required to arrive at a target-specific absolute estimate, because no specific target knowledge had to be generated in order to answer the comparative question. Put differently, from a selective accessibility perspective alone, implausible anchors should produce no anchoring effects at all. However, as research has shown, implausible anchors yield large and sometimes even larger anchoring effects than plausible anchors (e.g., Mussweiler \& Strack, 2001a; but see Wegener, Petty, \& Detweiler-Bedell, 2000 for an exception).

In order to fully explain the effects of implausible anchors, Mussweiler and Strack (2001a) therefore proposed an integration of their selective accessibility mechanism with an insufficient adjustment process (Tversky \& Kahneman, 1974; Jacowitz \& Kahneman, 1995; Quattrone, Lawrence, Warren, Souza-Silva, Finkel, \& Andrus, 1984). Specifically, during the comparative task, judges may compare the implausible anchor value to the upper or lower boundary of a distribution of plausible 
values they find plausible (see discussion above). This process would require judges to adjust from the implausible value to a self-set category boundary. However, simply adjusting the implausible anchor value to an acceptable boundary value would not provide judges with the specific target knowledge required to produce an absolute estimate. According to Mussweiler and Strack (2001a), judges solve the absolute judgment task by testing the hypothesis that the target is identical to the category boundary, which now serves as a new anchor value. Again, the positive hypothesis test would result in the selective activation of target-specific knowledge, which is then translated into an absolute estimate. Compared to the typical magnitude of absolute estimates, generated in response to considering plausible anchor values, absolute estimates in response to implausible anchors should be larger, since they are generated by testing the target's extension against the tail values of a distribution of plausible values.

Converging evidence from a number of studies appears to confirm this conceptualization. In one paper, Mussweiler \& Strack (2000a) have shown that responses to comparative questions are based on different types of knowledge, depending on the plausibility of the anchor value. As already described, solving the comparative question when anchors are plausible requires the generation of extensive exemplar knowledge (e.g., Mahatma Gandhi), whereas solving an implausible comparative question may simply involve knowledge about the general category to which the target belongs (e.g., human beings). A study investigating this logic showed that judges, asked to provide general category estimates (i.e., the length of the longest river on earth), answered this question more quickly after considering an 
implausible anchor during the comparative question. Judges asked to make specific target estimations (i.e., the length of the Mississippi river) responded faster after considering a plausible anchor value (Mussweiler \& Strack, 2000a).

In some studies, Mussweiler \& Strack (1997, 1999b, 2000a) were able to obtain differential response latencies to the comparative and absolute question, depending on the plausibility of the anchors. In studies varying the plausibility of the anchor values (e.g., Mussweiler \& Strack, 1999b), response latencies to the comparative question have been shown to be relatively long in the plausible anchor conditions, whereas absolute judgments were made relatively more quickly. The reverse pattern of results was obtained for implausible anchoring tasks. These results are consistent with the notion that the more elaborate hypothesis testing strategy occurs at different stages in the anchoring paradigm, depending on the plausibility of the anchor value.

To specifically examine the adjustment component of their model, Mussweiler and Strack (2001a) conducted two studies varying the plausibility of the anchor along with the extremity of the values in the implausible conditions. As reasoned by the authors, if judges indeed adjust the implausible anchor values to specific category boundaries, then two differentially extreme anchor values (valued in the same direction) should yield similar absolute estimates. One study anchored judges on the age of Gandhi at his death, the other on average temperatures in the Antarctic. Both studies showed the predicted pattern of similar mean estimates of Gandhi's age and the temperature in the Antarctic, regardless of the level of extremity of the 
implausible anchor value. As predicted, absolute estimates were larger in the implausible as compared to the plausible conditions.

Taken together, these findings seem to be supportive of the existence of a twostage process (adjustment plus selective accessibility) whereby judges solve implausible anchoring tasks.

\section{Summary}

The vast majority of current studies on the anchoring phenomenon seem to suggest that selectively activated semantics, generated in response to positively testing a hypothesis that the target is identical to the anchor value, theoretically mediate judges' responses to a subsequent absolute judgment question. As some studies show, changing the semantic context during the anchoring task eliminates or changes the anchoring effect. Similarly, evidence from lexical decision studies confirms the semantic activation notion of the model. Different types of knowledge (category versus exemplar) may be used to solve the comparative question, depending on the plausibility of the anchor. In addition to selective accessibility, an adjustment mechanism appears to underlie the effects of implausible anchors.

\section{Basic anchoring as an alternative to selective accessibility}

The overwhelming amount of evidence, seemingly in support of a selective accessibility (plus adjustment) notion of anchoring, appears in stark contrast to the simple, numeric priming explanation of anchoring ("basic anchoring"), proposed by Wilson, Houston, Etling, and Brekke (1996). According to Wilson et al.'s work, a short-term memory representation of the numerical anchor itself may be constructed 
during the comparative judgment phase. This short-term memory representation of a number, in turn, is hypothesized to directly influence responses to subsequent absolute judgments, without any of the semantic constraints on anchoring, predicted by the SA model. Contradictory to Mussweiler and Strack's model, Wilson et al. have shown anchoring effects, even when comparative and absolute judgment tasks were semantically unrelated. For example, in one study Wilson et al. asked one group of participants whether the number of nations in the UN is more or less than 1930 and another group whether the number of physicians in the local phonebook is more or less than 1930. In a subsequent absolute judgment task, all participants were asked to estimate the number of nations in the UN, showing anchoring effects with comparable effect sizes in both groups. This finding constitutes a puzzling opposition to the findings obtained, for example, by Strack and Mussweiler (1997) demonstrating the absence of an anchoring effect under conditions of changed judgmental dimensions (e.g., height versus width). If their selective accessibility process exclusively mediates all anchoring effects, then no anchoring effect should have been obtained in Wilson et al.'s study, since selectively activated knowledge regarding the number of physicians in the phonebook is irrelevant to an estimate of the number of African UN nations.

Noting this inconsistency, Wong and Kwong (2000) conducted a series of studies, demonstrating anchoring effects under conditions of changed targets between the comparative and absolute judgment, replicating Wilson et al.'s (1996) findings. Additionally, their design incorporated a variation of the typical anchoring paradigm by manipulating, between-subjects, the unit of measurement associated with the 
anchor values. Specifically, Wong and Kwong anchored participants on the length of the main runway of an airport in Hong Kong. In addition to receiving either a high or a low anchor value, the unit of measurement was varied such that half the participants received an anchor value in kilometer units and the other half in meter units. The anchors were chosen to be semantically identical, differing only in absolute numerical values (high anchors $=7300 \mathrm{~m}$ vs. $7.3 \mathrm{~km}$; low anchors $=2300 \mathrm{~m}$ versus $2.3 \mathrm{~km}$ ). The absolute question, for all participants, pertained to the price of a bus, changing both the target (from runway to bus) and the dimension of the judgment (from length to price). As predicted by Wilson et al.'s simple numeric priming hypothesis, the results showed the typical effect of considering a high versus low anchor on absolute judgments and an effect of unit on absolute judgments. Specifically, participants who received an anchor value of 7300 meters estimated, on average, the bus price to be higher than participants who received an anchor value of 7.3 kilometers. The same finding was obtained for the low anchor pair. As Wong and Kwong pointed out, these findings seem to undermine the selective accessibility theory of anchoring, because semantically equivalent anchors should have produced similar absolute estimates.

In response to these findings, Mussweiler and Strack (2001b) conducted two studies that specifically addressed Wong and Kwong's criticisms from a selective accessibility perspective. Further revising their work, Mussweiler and Strack (2001b) have recently argued that their model applies only to the kinds of anchoring effects initially proposed by Tversky and Kahneman (1974), or, to anchoring effects where semantics are allowed to operate on the absolute judgment. As they have argued, semantics can only operate in anchoring designs that hold targets constant between 
the comparative and absolute judgment. When targets change between the two tasks, pure numeric anchoring effects may still be obtained, as reported by Wilson et al. (1996) and by Wong and Kwong (2000). To provide evidence for this argument, Mussweiler and Strack (2001b) conducted a study, orthogonally varying anchor values (high, low), the judgmental target (same, different) and the unit of measurement associated with the anchor (e.g., m, km). For some participants, both judgments pertained to the height of a mountain, whereas for other participants, similar to Wong and Kwong's design, the target was changed to the number of languages spoken in the world. As in Wong and Kwong's study, high and low anchors differed only in terms of absolute numbers and were semantically equivalent. The results showed no effect of the semantically equivalent anchors (e.g., $1.6 \mathrm{~km}$ vs. $1,600 \mathrm{~m}$ ) when both judgments pertained to the same target, but showed a typical anchoring effect on height estimations of the mountain (e.g., 7.3km vs. 1.6km). Under conditions of changed targets, both the anchor value and the absolute value of the number, ignoring units, produced an anchoring effect on estimations of the number of world languages, replicating Wong and Kwong's (2000) findings.

\section{Summary}

The latest consensus in the literature on anchoring appears to be that a set of distinct psychological mechanisms may produce judgmental anchoring effects. The operation of these processes may be dependent on a) the type of anchor used (plausible versus implausible) and b) on the nature of the semantic context (e.g., targets changed or unchanged). The effects of plausible anchors are currently explained by a selective accessibility mechanism, involving the production of 
extensive exemplar knowledge. The effects of implausible anchors are explained by an adjustment plus selective accessibility process, involving the use of category and exemplar knowledge at different stages of the anchoring task. Finally, semantics appear to play absolutely no role in anchoring effects that involve changed judgmental targets and/or dimensions (with the exception of the contrast effect obtained in Strack \& Mussweiler, 1997, study 2). Instead, a simple numeric priming mechanism may be responsible for the production of anchoring effects under conditions of changed semantic contexts.

While Mussweiler and Strack provide a substantial amount of converging evidence in support of their model and for the importance of semantics in producing anchoring effects, a number of important questions regarding this effect remain. For instance, although it appears that specific target semantics play a role in producing the anchoring effect (e.g., Brandenburg gate example), anchoring effects have been demonstrated numerous times in situations when comparative and absolute question were semantically unrelated (e.g., runway/bus example). What might explain this inconsistency? Do the effects reported by Wong and Kwong (2000) or Wilson et al. (1996) simply belong to a subcategory of 'pure numeric' anchoring effects, or do semantics play a role in the mediation of these effects as well? If yes, what kind of semantics? Can a single mechanism account for semantic and numeric anchoring effects?

In the present dissertation project, I sought to answer these questions. In addition to arguing for an integration of semantic and numeric effects, I propose that implausible and plausible anchoring effects are attributable to the same general 
mechanism, thereby providing a more parsimonious account of the anchoring phenomenon. In the next section, I outline the specific features of this reconceptualization of the anchoring phenomenon.

\section{Anchor-based semantic priming: An integrative alternative}

A basic assumption of the present theory is that anchor values are mentally represented as words. These words semantically reflect the value of the anchor along a given judgmental dimension, considered by judges during the comparative judgment task. More specifically, it seems plausible that, while processing the comparative question, in which judges typically consider whether a target value is larger or smaller than a given anchor, judges are primed (e.g., Herr, Sherman, \& Fazio, 1983) with words related to the anchor value itself. Importantly, these words are hypothesized to reflect the numerical value of the anchor along a number of nonspecific semantic 'quantifiers' such as 'a lot', 'a little', 'long', short', 'high', 'low', 'expensive', inexpensive', which may vary in content as a function of a) the experimental design employed (anchor values high-low) and b) the judgmental dimension (e.g., length, height, weight, price) considered in connection with the category (e.g., mountains, animals, people), to which the target of the comparative question belongs. Via this simple priming mechanism judges are provided with a highly accessible, general, answer to the absolute question. Specifically, during the absolute estimation task, numerical estimates of the target's extension may be assimilated toward the primed semantic associate of the anchor value. Such a conceptualization, while clearly building on insights generated from the SA model, differs from the selective accessibility notion in that it assigns the semantic content 
used by judges to the anchor value itself and not to the target of the judgment. As a result, no positive hypothesis testing mechanism is assumed to be necessary for the solution of anchoring tasks, which allows for an explanation of the effects of plausible and implausible anchors, without differentiating between a 'selective accessibility' and 'adjustment' hypothesis. The specific details of this reconceptualization are discussed below, followed by a description of six studies that were conducted to explore it.

\section{The semantics of anchoring revisited}

One explanation of the anchoring effect comes from a perspective known as conversational relevance (Grice, 1975, discussed in Jacovitz \& Kahneman, 1995). For instance, judges relying on the maxim of quantity, discussed by Grice, might think that the experimenter in the study intentionally presented them with a numerical standard, possibly because the experimenter knows the answer to the absolute question. Based on this logic, judges might simply use the anchor value as a basis for their estimate, because they believe that the experimenter intended to provide them with a 'hint' as to the actual value. To rule out the effects of conversational relevance, many researchers (e.g., Strack \& Mussweiler, 1997) attempt to convince participants that the anchor values were chosen at random (e.g., by spinning a wheel of fortune, or rolling a set of weighted dice), which ought to render these anchor values, normatively speaking, uninformative with respect to any subsequent estimation task. The current consensus in the literature is that the 'random generation' solution virtually eliminates conversational relevance effects on anchoring. 
What appears to be somewhat of a puzzle is the fact that anchor values continue to influence absolute estimation tasks, despite the fact that judges likely believe they were randomly generated. As the following analysis will show, anchor values may robustly influence subsequent judgment tasks because researchers, of course, do not often randomly choose anchor values to present to their participants. Rather, they are specifically chosen by the researcher to represent high and low values with respect to a particular judgmental target or target category. In addition, numerical anchor values are not presented to judges in isolation, but are generally embedded in specific semantic contexts (see Wilson et al., 1996 for an exception). That is, whether randomly chosen or not, anchor values may prime associated concepts, thereby increasing the likelihood that these concepts will be used to answer the absolute question. A question, thus, arises as to the specific nature of these semantic associations.

Numerical values, considered in isolation, do not appear to be (semantically) quantifiable. For example, it would be difficult to state whether 200 is 'a lot' or 'a little' of something without knowing what that 'something' is. Of course, mathematically speaking, 200 is considered to be 'larger' than all of the numbers preceding it, and smaller than the numbers following it. However, in order to assign a semantic label reflecting its relative quantity, 200 would have to be appropriately contextualized. Within the anchoring paradigm, the comparative question provides the requisite context for the semantic labeling of the anchor value (see also Mussweiler \& Strack, 2000b). In connection with a judgmental target, such as the number of publications listed on a professor's vitae, the value 200 would be 
considered large, likely activating the semantic associate 'a lot' or words like 'productive', 'accomplished', and 'senior'. Conversely, embedded in a different context, such as the number of inhabitants of a city, 200 would be considered a small standard, increasing the activation of the semantic quantification 'low', 'small' or 'little'. Thus, the interpretation of a numeric value appears to depend on the contextual presentation of it, as does the direction of the semantic association activated in response to its consideration (e.g., high versus low) ${ }^{3}$.

The anchor value of 200 happens to have no specific judgmental dimension associated with it. Rather, it simply represents the numeric quantity of the two targets (number of publications and number of inhabitants). Many questions used in the anchoring literature, however, include more specific judgmental dimensions, such as the height or length of a target. The semantics associated with a particular anchor value may be mentally represented, in addition to such general semantics as 'a lot, or 'a little', in terms of a number of more specific judgmental dimensions. For example, a comparative question pertaining to the length of an airport runway may prime judges, depending on condition, with more specific concepts such as "long" and "short", whereas a question pertaining to the weight of a person might activate concepts such as "heavy" or "light". Thus, in addition to the direction of the semantic quantification, the specific content of the semantic prime may vary as a function of the judgmental dimension, considered by judges.

\footnotetext{
${ }^{3}$ In line with this argument, Mussweiler and Strack (2000b) have noted that the classification of a target as belonging to a specific category affects the plausibility of the anchor values. Additionally, target category membership may determine the range of values of subsequent absolute estimations. As they have shown, classifying an ambiguous target (e.g., "Xi Liang") as either a person or a cultural possession appears to affect the estimation of the target's age along numerical estimates implied by the two different categories. For example, estimates regarding the age of a cultural possession may vary by thousands of years, whereas the range of numerical estimates of a person's age is numerically much more restricted.
} 
Implicit in the foregoing discussion is the notion that numerical values are only semantically quantifiable as 'large' or 'small' in reference to, at least, the primary category to which the target of the judgment belongs. Unless, for example, the value 200 meters is referenced to a target, belonging to a known category, such as a television tower or an airport runway, it would be difficult to state whether 200 meters is "long" or "short", "high", or "low". A moment of reflection also reveals that specific target knowledge (i.e., specific knowledge about the television tower in Cologne or about Dulles Airport) may not be needed in order to classify the anchor value as 'a lot' or 'a little'. Rather, simply knowing that the Cologne Tower belongs to a category of 'television towers' would provide enough information for the classification of the value 200 meters as 'large', or 'high' (as far as towers go) or as "short", in connection with an airport runway. Thus, the present model assigns an important function to the target of the judgment, primarily in the sense that it provides information about the general category to which it is assigned at the time of the comparative judgment, thereby allowing for the semantic labeling of the anchor value. The procedure by which anchor values are selected may additionally support this notion. Plausibly high and low anchor values are typically chosen to be one standard deviation above or below a mean estimated value of the target feature, provided by an independent calibration group of judges (see Strack \& Mussweiler, 1997 for procedural details). Assuming a normal distribution of these estimates, high and low anchor values are also likely to constitute high and low values with respect to the general category, to which the target belongs (e.g., television towers). This fact further substantiates the notion that judges may be primed with general concepts 
related to the anchor value and may solve the absolute judgment task based on categorical rather than specific target knowledge.

It is, of course, possible for judges to know a lot more about the target of a given judgment than simply the category to which it belongs. In fact, anchoring effects have been demonstrated using expert real estate appraisers, who were shown to be influenced by differential price listings of the same piece of property (Northcraft \& Neale, 1987). According to the present model, however, specific target knowledge may not be necessary for anchoring effects to occur, whether the judge is expert or not. In the example above, the real estate appraisers could have simply noticed that the target belonged to an intermediate price category, within which the two anchor values constituted plausibly high and low standards. Thus, differential activation of the concepts "expensive" and "inexpensive" (for a house belonging to an intermediate price category), during the comparative judgment, may have directly influenced absolute appraisals of the property value, without specific reference to the features of the property. To avoid confusion, the present model does not stipulate that judges never recruit specific knowledge about the target from memory as part of the comparative judgment task. For example, judges who are motivated to process information accurately (e.g., Tetlock, 1983) may, perhaps ironically, compound the anchoring bias by recruiting extensive knowledge in support of a unidirectional hypothesis that the target is identical to the anchor value, much in line with predictions derived from the SA model. The primary goal of the present work is to show that anchoring effects can be obtained simply as a function of anchor-based semantics alone and that target-specific knowledge may not be necessary in the 
mediation of anchoring effects. If this analysis succeeds, it would provide the most general current framework for explaining a broad range of anchoring effects.

\section{Summary}

I've argued that the phenomenon of judgmental anchoring is a special case of a relatively simple priming process (e.g., Herr, Sherman, \& Fazio, 1983).

Specifically, comparing a given target to an anchor value may result in the activation of general semantic notions that are directly associated with the anchor value. The subsequent influence of such general concepts on absolute target estimates, according to my model, occurs as a function of a straightforward assimilation process, during which the ambiguous target of the judgment is categorized in terms of the primed semantic construct(s). The numerical range of the absolute estimation may depend on the categorization of a given target (Mussweiler \& Strack, 2000b). Note that this analysis is highly consistent with a large literature in social cognition demonstrating the influence of primed concepts on the interpretation of ambiguous targets. For example, in a well-known study by Higgins, Rholes, and Jones (1977) the ambiguous behavior of a target person "Donald" was interpreted in terms of two different traits (e.g., adventurous or reckless), depending on which of the two traits was primed in an earlier part of the study. Alternative concepts that were inapplicable to the ambiguous behavior of the target (e.g., listless) were not used to form an impression of the target. Thus, a target behavior appears to be assimilated to a category if the category can be applied to the interpretation of the target information. The current model proposes that anchoring constitutes a special case of this type of priming effect. 


\section{Effects of implausible anchors}

Recall from my previous description of the SA model that an initial theoretical disadvantage of the model may have been its inability to explain the effects of implausible anchors, using notions of a positive hypothesis test that results in the selective accessibility of target knowledge. As a consequence, an additional adjustment process had to be integrated with the existing selective accessibility notion, effectively explaining the effects of implausible anchors in terms of a twostage process.

While there is nothing inherently wrong with Mussweiler and Strack's (2001a) conceptualization, a general priming perspective may offer a more parsimonious alternative to their notions. From the perspective of the proposed model, the effects of implausible anchors can be explained in terms of the same mechanism, invoked to explain the effects of plausible anchors. Specifically, implausible anchor values are hypothesized to prime associated semantic concepts in the same way plausible anchor values do. However, implausible standards may prime more extreme semantic quantifiers than plausible standards do. Considering, for example, whether Gandhi was older or younger than 140 years when he died may prime judges with a concept 'very old', or simply increase the magnitude of the accessibility of the concept old, compared to the level of activation created in response to considering a plausible anchor value. Thus, judges may be primed with a, categorically speaking, more extreme answer, after comparisons with an implausible standard, which should result in more extreme absolute estimates of Gandhi's age. Such a mechanism also provides an explanation for the relatively stronger magnitude 
of anchoring effects, involving implausible standards, obtained in a number of studies (Mussweiler \& Strack, 1997; 1999b; 2001a).

This alternative conceptualization of the effects of implausible anchors does not assume that judges first adjust the implausible anchor toward a category boundary, before testing the hypothesis that Gandhi, for example, was of the age implied by the category boundary of human age. According to a general priming model, in response to receiving an implausible standard, judges may simply be primed with a generic concept (e.g., 'very old') that influences absolute estimates in terms of categorical notions of how old a 'very old' person is. The estimated value of the age of a 'very old' person, while likely to be close to the category boundary of human age, would not need to be generated by a deliberate adjustment process, according to my model. Put differently, judges may never actually consider whether Gandhi was 140 years of age and then adjust downward from that value. Rather, plausible and implausible anchors may simply prime differentially extreme semantic quantities within the category boundaries of age, for example.

According to this conceptualization, the magnitude of an absolute estimate, when influenced by an implausible standard, should be larger than those influenced by plausible anchor values. However, two implausible standards, differing in extremity in the same direction (e.g., 140 and 240 years of age), would not necessarily produce different absolute estimates as well. Recall, that Mussweiler and Strack (2001a) found that two differentially implausible standards of Gandhi's age produced similar absolute estimates, citing this finding as evidence of an adjustment process to the category boundary of 'human age'. From the standpoint of a semantic 
priming mechanism, both standards may have simply primed the concepts 'very old', without producing further semantic differentiations of the concept (e.g., 'very, very, very old'). Put differently, once different numerical standards are perceived as falling outside the range of plausible values for a target, they may simply prime similar associates.

In sum, implausible anchors are hypothesized to prime related semantic concepts, which produce anchoring effects. The primes are hypothesized to differ from those of plausible anchors in that they semantically reflect more extreme numeric values.

Critics of this notion might argue that a general priming explanation may not adequately explain the difference in response latencies to the comparative and absolute question, reported by Mussweiler and Strack (1997; 1999b), whereas a selective accessibility plus adjustment process does. As Mussweiler and Strack reported, in response to considering an implausible standard, participants answered the comparative question (whether the target's extension was higher or lower) relatively quickly, whereas responses to the absolute question were relatively longer. The opposite pattern of results was obtained when plausible standards were considered.

Regardless of the specific theoretical orientation adopted to explain the anchoring effect, there appears to be a general asymmetry between the two questions in the anchoring paradigm, depending on anchor plausibility. In fact, when asked to answer a comparative question involving plausible standards, judges may first generate an answer to the absolute question during the comparative judgment task. 
Put differently, in order to provide an answer to the question whether a given target's extension is higher or lower than a given (plausible) anchor value, judges may have to spontaneously generate an answer to the absolute question. As a result of this, an answer to the absolute question, following comparisons with plausible standards, is immediately available when judges are presented with the absolute question, accounting for the quick response times to that question (relative to the comparative judgment task). When the anchor values chosen for a comparative question are implausible, on the other hand, judges are able to immediately provide an answer to the comparative question, without first generating an absolute estimate of the target's extension. Thus, when asked to provide an absolute estimate following a comparison task involving implausible anchors, judges may still have to generate the answer to the absolute question. The point here is that differential response latencies, as a function of anchor plausibility, are not unequivocally indicative of the operation of the psychological processes proposed by Mussweiler and Strack. Thus, the asymmetry in response times may be a function of a number of mental processes and not exclusively reflective of the operation of a hypothesis testing or adjustment process.

\section{Generality of the anchoring effect}

From a general, anchor-based semantic priming perspective, judgmental anchoring effects are not merely limited to the anchoring paradigm proposed by Tversky \& Kahneman, 1974, in which the target of the comparative question is identical to the target of the absolute question. In fact, another advantage of a nonspecific, anchor-based semantic priming theory of anchoring lies in its potential to 
explain a wide range of effects involving changed targets and/or dimensions between the comparative and absolute question. For example, the same semantic concept (e.g., 'old') may mean different things, yielding different absolute estimates, depending on the target category of the absolute judgment. Thus, a concept, such as 'old' may be initially primed in response to considering a person's age, yet may generally influence a variety of age-related absolute estimations (e.g., the age of an object). Put differently, while the activation of anchor-based semantics is clearly dependent on the judgmental context of the comparative question, the subsequent use of the semantic prime may be relatively independent of the context in which it was activated. At the same time, the general priming hypothesis includes the potential to explain the absence of anchoring effects involving changed judgmental targets, which have been previously reported as well (e.g., Strack \& Mussweiler, 1997). For example, after being primed with the concept 'old', in response to considering a high standard of a person's age, a subsequent estimation of the price of an object may be unaffected by the 'old' prime, because the person does not associate the concept 'old' with 'expensive'. In other words, concepts primed in response to processing the anchor value in a comparative question, may not be indiscriminately relevant to all subsequent absolute estimations, consistent with the relevance notion of activated knowledge in much of the social cognitive priming literature (see Higgins, 1996 for a review).

However, it is also possible for initially primed concepts, such as 'old' to associatively bring to mind other concepts, such as 'expensive', depending on whether judges strongly associate those two concepts. Thus, from the current 
perspective, the presence or absence of anchoring effects, as well as the nature of the effect (assimilation versus contrast) under conditions of changed semantic contexts, may vary as a function of a) the degree to which anchor-based semantic concepts are associatively linked to other concepts in the judge's mind (cf. Anderson \& Bower, 1973; Bower, 1981; Meyer \& Schvaneveldt, 1971; Srull \& Wyer, 1979, 1980), and b) as a function of factors related to the judged usability (Higgins, 1996) of information. I will consider these aspects in turn.

\section{Rule-based linkages among concepts}

The present analysis is based on the notion that the consideration of an anchor value, within a given context, increases the accessibility of concepts related to the anchor value itself, which may subsequently affect absolute target estimates, resulting in anchoring effects. Specifically, the target, in a judge's mind, may be meaningfully evaluated in terms of purely anchor-based semantics, such as "rich", "old", "productive", "expensive" and so forth, depending on the context of the judgment. From a social information processing perspective, it is also plausible that concepts originally primed in response to answering a comparative question, may associatively bring to mind other concepts. As Bower (1981) put it,

"The contents of consciousness are the sensations, concepts, and propositions whose current activation level exceeds some threshold. Activation presumably spreads from one concept to another, by associative linkages between them." (Bower, 1981, p. 134). 
Applied to the current discussion, the initial consideration of an anchor value in one context might activate associated concepts, which are subsequently relevant to the evaluation of different targets, involving different judgmental dimensions.

To simplify the discussion of this possibility, I will define such associative knowledge activation processes in terms of associative rules of an "IF-THEN" variety (Pierro, Mannetti, Kruglanski, \& Sleeth-Keppler, 2004; Holyoak, Koh, \& Nisbett, 1989). Simply put, thinking about a concept X may lead one to also think about concept $\mathrm{Y}$ because a condition exists in the judge's mind that links the two concepts together. The relative nature and strength of any given associative rule between two concepts generally depends on a person's subjective experience with the world. Specifically, I assume that persons generate associative rules of the "IF-THEN" variety between two concepts (or stimuli in general) because the two concepts share rule-relevant features. For example, persons with different occupations might learn to conditionally link concepts that reflect separate judgmental dimensions, based on their interactions with vocationally relevant stimuli. Thus, a person in the business of selling cellular phones might, over time, generate a rule that links the concept "expensive" with the concept "light" or "small", because expensive cellular phones tend to be lighter (and smaller) than less expensive models. Similarly, a person working for a delivery company might link the concept "fast" with the concept "expensive" because faster delivery service is invariably more expensive than slower service, in the delivery business. Persons working with diamonds might develop rules such as "if heavy, then expensive", or "if clear, then valuable", and so forth. 
Applied to the current topic, whether or not anchoring effects occur under conditions of changed judgmental dimensions may be critically dependent on the operation or rule-based associative processes, in response to processing a given comparative question. Take, again, the example of the person in the business of selling cellular phones. He or she might be asked to consider whether the price of a given item is higher or lower than either a high or a low standard of the value of the object. Thus, depending on condition, the person may be primed with either the concept "expensive" or "inexpensive". Subsequently, after considering a comparative question involving the price of an object, an anchoring effect may be obtained on an absolute question involving the weight of a completely unrelated object. Specifically, in the case of the activation of a rule "if expensive then light", or "if inexpensive, then heavy", anchoring effects may be obtained on an ambiguous weight estimate, after considering the price of an object. In the example above, the weight of the unrelated object should be judged as higher in the "inexpensive" comparison condition and as lower in the "expensive" comparison condition, depending on the rule that is accessible at the time of the absolute judgment.

Such a conceptualization also provides a straightforward explanation of contrast effects, which are sometimes obtained in judgmental anchoring. In the traditional anchoring paradigm introduced by Tversky \& Kahneman (1974), one typically expects to find assimilative anchoring, that is, absolute estimates within the general direction of the anchor. Research has shown, however, that judges sometimes contrast their absolute estimates away from the standard (or anchor) considered in an earlier comparison task (e.g., Strack \& Mussweiler, 1997, study 2). While one 
explanation for contrast effects is based on meta-cognitive correction processes (e.g., Martin \& Achee, 1992; Strack \& Mussweiler, 1997, discussed in the next section), another explanation may be based on the possibility that certain associative rules may change the direction of the absolute estimates away from the initial anchor value. Thus, rules, conditionally linking two separate judgmental dimensions, may differ with respect to the directional relation between two 'semantic values' on those dimensions. In the cellular phone example above, the nature of the rule is such that a "high" concept (expensive) associatively brings to mind a "low" concept (light, small). Hence, in response to considering a high anchor value of price, a contrast effect may be obtained on a subsequent weight or size estimation task, provided an appropriate rule is sufficiently accessible and used during the judgmental task. Similarly, assimilation effects should be obtained under conditions of changed judgmental dimensions, when an associative rule links two "high" values on two separate judgmental dimensions, such as "if expensive, then large". These possibilities add to the current literature on anchoring by providing a general framework for the explanation of anchoring effects under conditions of changed judgmental dimensions. Specific associative rules are hypothesized to determine a) when anchoring effects occur under conditions of changed judgmental dimensions and b) what direction the effect will take (contrast versus assimilation).

\section{Correction processes}

An absence of anchoring effects under conditions of changed dimensions, as well as contrast effects, can also be explained by the perceived irrelevance of activated semantic notions, such as "high", for an estimation task involving, for 
example, the "width" of a target. There exists substantial evidence that people make judgments concerning the relevance or appropriateness of mentally activated information for a response, which in turn can determine their use of this information (see Higgins, 1996 for a review of the literature on judged usability). Thus, it may be possible for judges to actively inhibit certain pieces of knowledge if they judge them as inappropriate for a judgment. For example, Strack and Mussweiler (1997), explained the absence of an anchoring effect (e.g., Brandenburg Gate study) by referring to the inapplicability of activated target semantics to a judgment involving a changed dimension. Similarly, from a general priming perspective, the concept 'high', primed during the comparative task, may have been judged as irrelevant for the estimation of the gate's width.

Additionally, the perceived change in dimension itself, between the two questions, could have signaled to participants, that any knowledge activated during the first question, should not be used to answer the second question.

This analysis implies that judges who have sufficient capacity (and motivation) to process information may inhibit primed semantic concepts more often, when they perceive a change between the comparative and absolute judgment or if they perceive activated knowledge as irrelevant for a judgment. Conversely, judges low in motivation or low in cognitive capacity may assimilate a judgment to a prime, because they may fail to perceive the change in questions or, because they are less able to make informational relevance judgments.

Similarly, when judges perceive a change in judgmental dimensions, contrast effects may occur on absolute estimates because of meta-cognitive over-correction for 
the influence of the concepts primed in response to considering the comparative question. Strack and Mussweiler (1997) invoked this possibility to explain the contrast effect obtained in the Antarctica-Hawaii temperature study and would also apply to the general priming model proposed herein. 


\section{The Present Research}

The foregoing analysis may be summarized in terms of a number of testable implications.

1) Judgmental anchoring effects, involving plausible and implausible anchors, are hypothesized to result as a function a simple semantic priming mechanism. Specifically, when judges consider a numerical anchor value within the context of a

given comparative judgment, they are primed with words related to the anchor value. These anchor-associated concepts are hypothesized to directly influence people's subsequent absolute target estimations. Studies 1 and 2 were designed to test these notions.

2) Once people are primed with anchor-related notions in response to processing a given comparative question, they may (associatively) think of additional semantic concepts that may influence a variety of subsequent target estimations. Thus, depending on the nature of these associations (or rules) the influence of the anchor value may 'spread' to judgmental targets not specifically considered during the comparative judgment task. Study 3 tested this implication of the general semantic priming notion of anchoring.

3) Study 4 was designed to test the notion that correction processes may affect the extent to which anchoring effects are obtained under varying conditions of the anchoring paradigm. Depending on sufficient levels of cognitive capacity, judges may inhibit knowledge after perceiving an illegitimate change in judgmental dimensions or targets, reducing the anchoring effect. This implication also allowed the present 
study to address an existing critical evaluation (Mussweiler \& Strack, 2000b, study 3)

of the idea that general, anchor-based semantics may be responsible for producing anchoring effects.

4) If general, anchor-based, semantics produce anchoring effects, then considering a numerical value (e.g., 45) ought to prime different associated concepts, depending on the target of the judgment. Put differently, instead of varying the numerical values of the anchors as part of the comparative question, one might obtain anchoring effects by holding the numerical anchor constant and varying the target of the judgment along the same judgmental dimension. Studies 5 and 6 in this series were designed to test this notion, providing perhaps the most direct test of the anchorbased priming hypothesis.

\section{Studv 1}

The first study in this series combined the standard anchoring paradigm with a lexical decision task (Meyer \& Schvaneveld, 1971, see Neely, 1991 for a review). In this task, participants read a string of letters and attempt to identify, as quickly as possible, whether it is a word or a nonword. Reaction times for words are quicker if a context that is related to the target word has been provided. For example, participants recognize nurse as a word more quickly if they have just read the word doctor than if they have just read an unrelated word, such as bread.

The lexical decision task was combined with the standard anchoring paradigm in order to assess the degree to which persons think of anchor-consistent words after receiving the comparative question. For example, according to the anchor-based priming model described above, participants are hypothesized to be primed with 
words related to the concept 'old' in response to receiving a comparative question including a high standard of a target person's age. This should be reflected in faster lexical decisions for words associated with the semantic category 'old'. Conversely, asking participants to compare a target person's age to a low standard should facilitate lexical decisions for words associated with young age. Thus, one purpose of the present study was to test the extent to which comparing targets to an anchor results in the increased accessibility of words related to the anchor values themselves, providing evidence that such semantic concepts may mediate anchoring effects.

A second, and more important, purpose of this study was to test whether the effects of implausible anchors are a function of the same process, assumed to underlie the effects of plausible anchors. Specifically, I expected an increased facilitation of response latencies to anchor-consistent target words when judges consider implausible anchor values as standards (compared to plausible anchor values). Consistent with the notion that implausible anchor values increase the accessibility of anchor-consistent words, relative to plausible anchors (see introduction), I also expected implausible anchors to yield stronger anchoring effects, compared to the effects of plausible anchors, replicating previous research on implausible anchors (e.g., Mussweiler \& Strack, 2001a).

\section{Method}

Participants. 111 (male and female) University of Maryland students enrolled in an introductory psychology course participated for course credit.

Materials. The present research was closely fashioned after the experimental paradigm employed by Mussweiler and Strack (2000a, studies $1 \& 2$ ). All materials 
were presented to participants on IBM compatible computers, using the MediaLab ${ }^{\mathrm{TM}}$ software package. Consistent with the classic anchoring paradigm, participants received question pairs including a comparative and an absolute question. Specifically, for each question pair, participants first indicated whether the numerical value of a target was higher or lower than a given anchor value before being asked to give an exact absolute estimate of the numerical value. All participants first received five identical question pairs, to provide a believable context for the cover story, described below. The sixth question pair included the independent variable for which the anchor values were varied. Hence, roughly one-fourth of the participants received a high plausible anchor value during the comparative question, one-fourth a low plausible anchor value, one-fourth a high implausible anchor value and one-fourth a low implausible anchor value. As part of the critical comparative question, participants were asked whether Mahatma Gandhi was older or younger than either 79 or 64 years for the plausible conditions, and older or younger than 140 or 9 years for the implausible conditions. The order of the questions was held constant.

Four different categories of letter strings were used in the lexical decision task: words associated with old age (such as "old", "ancient", "mature"); words associated with young age (such as "young", "youth", "infant”); neutral words, such as pencil, cow, hair, and zebra; and nonwords (see Table 1).

In sum, study 1 is based on a 2 (high vs. low anchor) X 2 (plausible vs. implausible) X 2 (lexical decision for old vs. young words) mixed factorial design. The first two factors were manipulated between participants and the third factor within participants. 
Table 1

Letter strings used in the lexical decision task (Studies $1 \& 6$ )

Word type

Letter string

Old words

Young words

Neutral words

Nonwords old, elderly, aged, adult, ancient, mature, senior young, youth, child, baby, infant, immature, junior eat, shoe, devil, sad, pencil, fall, hair, sobering, water, car, jacket, walk, clean, cow, giraffe, trunk, ice, horn, buffalo, horse, camel, house, zebra, rain, dog, loan, tiger, lake, monkey, table, coyote, gazelle, lion, cat phrup, vapse, dwighst, thaips, clulths, tweivvs, fenth, skrunn, cowce, trebe, stromn, thwawz, tarb, swardes, crolt, croiced, gruiggs, gwoints, fliegue, demb, streeved, dweigues, soys, kwaughpth, phleused, blulcs, trawvs, tempced, wroughgnth, brepth, teene, tovs, flurld, skwoizzed, wrintse, creussed, swylch, shrighte, flane, snygues, plail, brighvs, sckwoach, gyte, dourck, skoal, sckwerthe

Procedure. Participants were run in groups of 4-6, with each participant seated at a PC workstation. Upon arrival participants were informed that they were taking part in the construction of a general knowledge questionnaire. The study, participants were told, included a comparison of traditional methods that use general knowledge questions, with more modern methods that analyze how quickly and accurately people recognize words. It was emphasized that the purpose of the study was to improve the wording of general knowledge questions. Moreover, participants were informed that some of the questions required a comparison with a given 
standard and that these standards had been randomly selected by using a mechanism similar to that of a wheel of fortune. It was pointed out that this was necessary to minimize the potential influence the standards may have on the answers and to identify the impact of different question formats. The emphasis of the random selection procedure was designed to reduce the informational value of the anchors through conversational inferences (Grice, 1975) ${ }^{4}$.

After receiving instruction regarding the use of a computer keyboard, participants were instructed to answer the comparative question by pressing appropriate keys, corresponding to the two possible answers to the question (higher or lower). To provide answers to the absolute question, participants were instructed to use the number pad on the keyboard. Finally, participants were told to answer accurately, but to move quickly through the questions.

Following the instruction phase, five pairs of general knowledge questions were presented to participants. The lexical decisions were assessed after responses to the sixth comparative question had been made. Two similar studies conducted by Strack and Mussweiler (2000a), using a lexical decision task, found that the order of the task within the anchoring paradigm did not affect response latencies. However, consistent with the theoretical assumption that anchor-consistent words theoretically mediate the anchoring effect, lexical decisions were assessed immediately after the comparative task.

Thus, after responding to the sixth and critical comparison question, participants were told that the next part of the experiment consisted of a new method to assess the wording of general knowledge questions. They were told that this

\footnotetext{
${ }^{4}$ The same methodological precaution was taken in all subsequent studies as well.
} 
method was designed to implicitly assess general knowledge by analyzing how quickly people discriminate words from nonwords. It was pointed out that letter strings would be presented on the computer screen and that the participants' task would be to indicate whether these strings constitute words or nonwords. They were instructed to provide the appropriate answer, using keys on the keyboard. Participants were told to press the "y" key as quickly as possible if a letter-string is a word and the "n" key as quickly as possible if it is a nonword. All participants were told to position their forefingers on the two keys before the word appeared on the screen.

The letter-strings were presented after the appearance of a focus point in the middle of the screen. The focus point was displayed for $400 \mathrm{~ms}$, followed by a lettern string that remained on the screen until an answer key had been pressed. After a pause of 3 seconds, the next string of letters appeared.

The task included 10 letter strings as part of a practice trial. The 11th through final trials were the critical trials, including 'old' and 'young' words, neutral and nonwords for a total of 96 letter strings. The stimulus set contained an equal number of words and nonwords. The order of presentation was completely randomized.

After the completion of the lexical decision task, the final absolute question was presented. Specifically, participants were asked to provide an absolute estimate of Gandhi's age. Finally, all participants were debriefed, thanked and dismissed.

\section{Results}

Absolute estimates. Consistent with predictions, the anchoring effect was replicated. Participants provided higher estimates of Gandhi's age after being exposed to a high anchor compared to a low anchor of his age, $F(1,107)=26.889, p<.001$. 
Additionally, an Anchor (high vs. low) X Plausibility (high vs. low) analysis of variance (ANOVA) yielded the predicted interaction, $F(1,107)=5.017, p<.05$. As the inspection of table 2 reveals, consideration of implausible anchor values yielded an overall larger effect, compared to the plausible anchor condition.

Table 2

Absolute estimates of Gandhi's age by anchor and plausibility (Study 1).

\begin{tabular}{ccc}
\multicolumn{2}{c}{ Plausibility } \\
\hline High & Low \\
\hline
\end{tabular}

\begin{tabular}{ccccc} 
Anchor & M & SD & M & SD \\
\hline High & 81.40 & 11.20 & 88.21 & 17.75 \\
Low & 72.47 & 14.73 & 65.48 & 18.75 \\
\hline
\end{tabular}

Lexical decisions. Following recommendations by Fazio (1990), I first transformed all individual reaction times using a natural log transformation, in order to minimize the influence of outliers. Furthermore, in order to control for individual differences in response times, I calculated a baseline response index by averaging participants' responses to the nonwords and neutral words. Subsequently, for each participant, this baseline response index was subtracted from the average response to the old and young words, respectively. Thus, all subsequent analyses were performed using deviation indexes from the participants' individual baseline of responding to the noncritical words and nonwords. Negative values represent responses that are faster than this baseline; positive values represent responses that are slower. 
As can be seen in table 3, the speed with which participants identified the old and the young words in the lexical decision task clearly depended on whether they received a high versus low anchor and whether the anchor value was plausible or implausible. In particular, participants in the high-plausible anchor condition responded faster to words related to the general dimension 'old', whereas participants in the low-plausible anchor condition were faster at recognizing words related to the concept 'young', empirically replicating earlier work by Mussweiler and Strack (2000a, studies 1 \& 2).

Table 3

Response latencies for lexical decisions for old and young words by anchor and plausibility (Study1).

Plausibility

\begin{tabular}{cccccc} 
& \multicolumn{2}{c}{ High } & & \multicolumn{2}{c}{ Low } \\
\cline { 2 - 3 } \cline { 6 - 6 } & Old word & Young word & & Old word & Young word \\
Anchor & $\mathrm{M}(\mathrm{SD})$ & $\mathrm{M}(\mathrm{SD})$ & & $\mathrm{M}(\mathrm{SD})$ & $\mathrm{M}(\mathrm{SD})$ \\
\hline \multirow{2}{*}{ High } & $-.10(.08)$ & $-.09(.08)$ & & $-.13(.08)$ & $-.08(.08)$ \\
Low & $-.08(.11)$ & $-.13(.07)$ & & $-.07(.11)$ & $-.15(.10)$ \\
\hline
\end{tabular}

Note. Deviations from the baseline speed of responding to neutral and nonwords (in $\log \mathrm{ms}$ ) are reported. Lower values indicate faster responses.

Secondly, and more importantly, results showed an overall stronger activation pattern in the implausible (vs. plausible) anchor conditions. That is, participants were significantly faster at recognizing "old" target words in the high-implausible anchor condition, compared to the high-plausible anchor condition. Similarly, participants were faster at recognizing "young" target words in the low-implausible condition, compared to the low-plausible condition. Thus, of greatest theoretical interest was the overall Anchor (high vs. low) X Plausibility (high vs. low) X Target (old vs. young 
words) mixed-model analysis of variance (ANOVA), which yielded a significant three-way interaction effect, $F(1,107)=6.370, p<.05$. In addition, this analysis yielded a significant two-way interaction effect, namely between Anchor (high vs. low) and Target (old vs. young words), $F(1,107)=26.118, p<.001, M^{5}$ (anchor high, old words $)=-.112, M($ anchor high, young words $)=-.0894,, M($ anchor low, old words $)=-.0748, M$ (anchor low, young words $)=-.140$. None of the other effects approached significance.

\section{Discussion}

The results of the first study showed that answering a comparative question about a person's age activated semantic notions related to the anchor values themselves. Specifically, participants were faster at recognizing words related to the concept 'young' in a lexical decision task after being presented with a low anchor of Gandhi' age, and faster at recognizing words related to the concept 'old' in the high age anchor conditions.

The lexical decision results, in the four plausible anchoring cells of this study, empirically replicate the lexical decision data obtained in studies by Mussweiler and Strack (2000a, studies $1 \& 2$ ), which showed, for example, that words related to either high or low temperatures (e.g., sun, beach, or snow, sleigh) were recognized faster, depending on whether participants received a high versus low anchor of the mean annual temperature in Germany. It should be noted here that, although the SA model proposes that specific exemplar knowledge (i.e., specific knowledge that supports the contention that the mean annual temperature in Germany is either high or low), this

\footnotetext{
${ }^{5}$ Note: Estimated marginal means are reported here.
} 
notion was not unequivocally supported by the results obtained by Mussweiler and Strack (2000a, study 1), because the target words could equally well reflect general semantic notions related to the anchor values themselves (e.g., sun, beach and hot are words generally indicative of high temperatures). However, the lexical decision results obtained by Mussweiler and Strack (2000a) are readily interpretable in terms of the general priming mechanism outlined in this dissertation.

In addition to replicating the results obtained by Mussweiler and Strack (2000a) in the plausible anchoring conditions, results showed that lexical decisions for words related to the dimensions old and young depended not only on the anchor values (high vs. low) but also on the plausibility of the anchor value (plausible vs. implausible). Specifically, comparing a target to implausibly high and low anchor values resulted in an increase in accessibility of general, anchor-based semantics, relative to the level of activation reflected in response latencies after comparisons with plausible anchor values. Importantly, these differences in response latencies of anchor-based semantics, as a function of the plausibility and the anchor values themselves, map on to the stronger anchoring effect obtained in the implausible vs. plausible anchoring conditions. To my knowledge, this is the first study in the anchoring literature that measured the accessibility of anchor-related words in an implausible anchoring condition.

Thus, from a general priming perspective, the explanation of plausible anchoring effects as well as implausible anchoring effects appears to involve the same anchor-based semantic priming mechanism that appears to result in differential levels 
of accessibility of anchor-related words as a function of the anchor value and the plausibility of the anchor.

To provide convergent support for a general priming model of anchoring effects, the second study was designed to 'bypass' the comparative question stage of the anchoring paradigm by priming anchor-related semantic concepts directly, using a priming technique. Specifically, participants in the second study were, depending on condition, primed with the concept 'long' or 'short', while working on a lexical decision task. Instead of using the lexical decision task to measure semantic activation, I modified it to prime semantic categories that were hypothesized to mediate anchoring effects in the domain of length judgments. Afterwards, participants were asked to estimate the average length of a whale. Theoretically speaking, priming anchor-related words directly should constitute the equivalent of presenting an actual comparative question and thus, should produce anchoring effects similar to those obtained in a standard anchoring paradigm.

\section{Study 2}

\section{Method}

Participant and Design. 31 (male and female) students at the University of Maryland participated in exchange for course credit in an introductory psychology course. Participants were randomly assigned to the three cells ('long' prime vs. 'short' prime, vs. control condition) of a single factor between-subjects design. Procedure and Materials. Participants were run in groups of 2-6, with each participant seated separately at a PC workstation. The priming manipulations, 
including all related instructions and stimulus materials were presented to participants using the MediaLab ${ }^{T M}$ experiment software package. Participants took part in what was ostensibly a pretest for the construction of a general knowledge survey. Participants were informed that they would first respond to a short version of a novel test of general knowledge, during which they had to decide as quickly as possible, whether a string of letters presented to them on the computer screen was a word or not. Following this task, participants were instructed to answer a number of general knowledge questions.

Priming manipulation. I used a lexical decision task to prime the semantic categories long and short. The specific details related to the presentation of the letterstrings are identical to those described in study 1 with the following exception: depending on condition, the words presented to participants consisted either of words related to the semantic category "long" (long, elongated, stretched, extended, expanded, lengthy, expansive) or "short" (short, puny, dwarfed, contracted, compact, stunted, small). Both groups of participants also received an equal number of nonwords (e.g., ploced, soys, teene). Thus, depending on condition, participants were exposed to words related to the semantic category long or short, while ostensibly working on a novel test of general knowledge involving the ability to distinguish words from nonwords. Participants in the control condition did not respond to the lexical decision task and directly responded to the absolute question.

Absolute question. After completing the lexical decision task, participants were told that a separate part of the study was designed to pretest the wording for a number of general knowledge questions. All participants received a number of 
absolute questions on a questionnaire, asking them to estimate quantities related to a number of different targets. The first question on the list was comprised of the critical dependent variable and asked participants to estimate the average length of a whale (in feet). Following this question, participants responded to 25 filler questions. These questions were included to make the cover story regarding the construction of a knowledge survey believable.

Suspicion check. In order to assess whether participants ${ }^{6}$ were aware of the connection between the priming task and the absolute estimates they provided, I used a funneled debriefing procedure suggested by Bargh and Chartrand (2000). None of the participants reported any suspicion that the lexical decision task may have influenced their responses to the subsequent questionnaire. Finally, all participants were debriefed, thanked and dismissed.

\section{Results and Discussion}

As depicted in Figure 1, participants in the "long" priming condition gave higher estimates of the average length of a whale $(M=91.4, S D=49.6)$ compared to participants in the "short" priming $(M=45.36, S D=37.41)$ and "control" $(M=49.8$, $S D=26.25)$ conditions. An overall one-way analysis of variance (ANOVA) revealed a statistically significant effect, $F(2,28)=4.355, p<.05$. A subsequent analysis (Dunnett's t) revealed that the difference between the "long" priming condition vs. control condition was statistically significant, $p<.05$, whereas the "short" priming vs. control difference was not, $p>.05$. Furthermore, an effect size calculation revealed

\footnotetext{
${ }^{6}$ Only participants in the two priming conditions were checked for suspicion.
} 
that the effect is comparable in size to anchoring effects obtained in prior research, $r^{2}=.24$, (see Wilson et al, 1996).

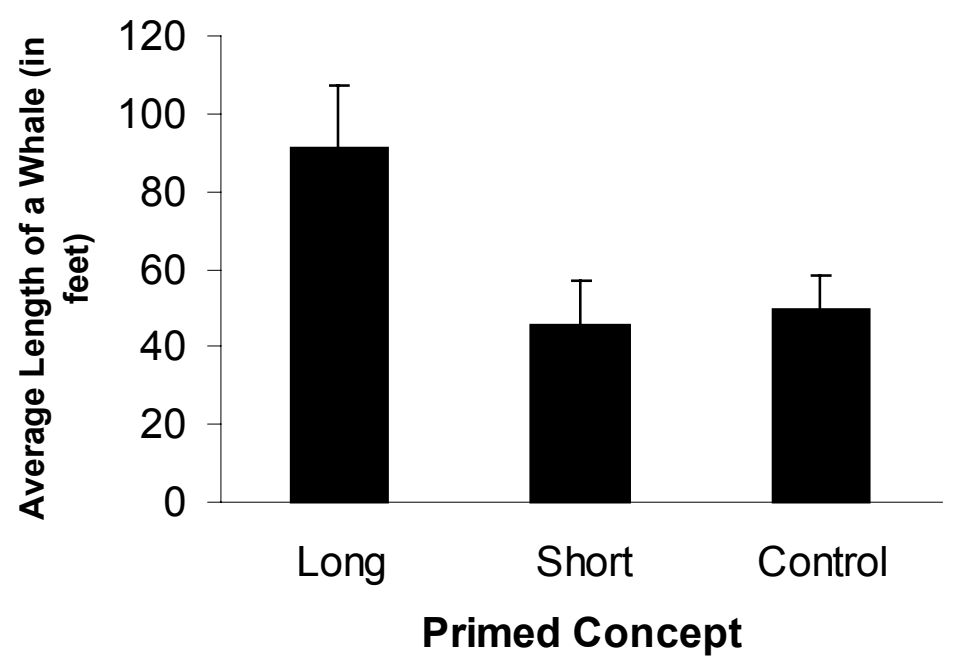

Figure 1. Absolute estimates by priming condition (study 2).

These results provide convergent support for the notion that semantic concepts, reflecting general, numerical quantities, influence absolute estimation tasks in judgmental anchoring paradigms. Specifically, this study was designed to bypass a comparative judgment task involving the length of a whale and prime concepts similar to those that would have been activated after considering a high or low anchor value of the length of this target. Interestingly, despite the fact that the semantic categories primed during the lexical decision task were not referenced to a specific target (in fact, the lexical decision task appeared disconnected to participants from the subsequent estimation task), the estimation of the length of a whale was strongly influenced by these general semantic notions. Thus, this study, again, supports the analysis that anchoring effects may be mediated by a simple priming mechanism. 
To further test these notions and to provide an explanation for anchoring effects under conditions of changed targets/dimensions, between the comparative and absolute question, a third study in this dissertation project was designed to demonstrate anchoring as a function of semantic concepts that are associatively linked via a conditional rule (see implication 2 of the present model). Recall that the existing two major models of anchoring predict that anchoring effects under changed semantic conditions are either driven by purely numerical representations of the anchor values (Wilson et al., 1996), or result in a contrast effect (Mussweiler \& Strack, 1997, study 2), due to meta-cognitive considerations. Based on the current model, concepts primed in response to considering an anchor value during the comparative question might influence a seemingly unrelated absolute estimation (e.g., involving a different target and a different judgmental dimension), through the rulebased activation of associated concepts, judgmentally relevant with respect to the changed target of the absolute question. This possibility was directly tested in study 3 .

Study 3 consisted of a rule-priming task, followed by an anchoring task during which the target of the judgment changed between the comparative and absolute question. Specifically, in one condition of the third study, I first primed participants with a rule that "if expensive, then heavy" and "if inexpensive, then light", using a questionnaire-based manipulation. Since both 'heavy' and 'expensive' (inexpensive, light) are denotative of 'high' (or 'low') values along these two dimensions, this condition was designed to result in an assimilation effect during a subsequent anchoring task. In the second condition, I primed the opposite rule: "if expensive, then light" and "if inexpensive, then heavy". These two conditions linked a high 
semantic value to a low semantic value (and vice versa), which was predicted to result in a contrast effect. Subsequently, judges received a comparative question involving either a high or a low anchor of the price of an object, presumably priming either the concepts 'expensive' or 'inexpensive'. After answering the comparative question, judges were asked to provide an absolute estimate of the weight of an object (the dependent variable). To restate, the direction of the anchoring effect, with respect to the weight of the target, was predicted to vary as a function of the rules primed during the first part of the study.

\section{Study 3}

\section{Method}

Participants and Design. 43 (male and female) students at the University of Maryland participated in exchange for course credit in an introductory psychology course. Participants were randomly assigned to the four cells of a 2 (high vs. low anchor) X 2 ('expensive $=$ heavy'/'inexpensive $=$ light' rules vs. 'expensive $=$ light'/'inexpensive = heavy' rules) factorial design.

Procedure and Materials. Participants were run in groups of 2-4. Each participant received a packet of surveys, including general instructions similar to those provided in studies 1 and 2. Following these general instructions, participants received a questionnaire entitled 'personal knowledge' (see Appendix). This questionnaire contained an adapted version of a task, designed by Erb, Fishbach, \& Kruglanski (2002) to manipulate associations between semantic terms, presumed to activate a general rule (e.g., "small is likable" or "big is likable"). As part of this task, 
participants were asked to answer various questions (e.g., "what is your opinion of George Bush? What is the size of a swordfish?), by recording their responses on one of two scales: one ranging between the "like" and "dislike" ends (appropriate for the question about Bush), the other ranging between the "small" and "large" ends (appropriate for the question about the swordfish). In one condition, "small" and "like" anchors were placed at the same end of the scale and "big" and "dislike" on the other, thus creating an association between the terms and presumably activating the "small is likable" and "big is dislikable" rules. In another condition, these terms" concordance was reversed, hence activating the "large is likable" and "small is dislikable" rules. Erb et al. (2002) found that participants exposed to the "small is likable" and "big is dislikable" rules reported a greater preference for a small school in a message describing its virtues than participants exposed to the opposite rules.

Using this specific methodology, for half the participants in this study, an 'expensive $=$ heavy' and 'inexpensive $=$ light' rule was created, whereas for the other half, the opposite rule-pair was created ('expensive $=$ light'; 'inexpensive $=$ heavy'). Afterwards, participants received a comparative question, on a separate questionnaire, asking them to indicate whether the average price of a wristwatch was higher or lower than $\$ 10,000$ in the high anchor condition and $\$ 2$ in the low anchor condition ${ }^{7}$. A group of participants ( $\mathrm{N}=26)$ who participated in a pretest, and only provided absolute estimates related to a number of targets, spontaneously thought the average price of a wristwatch was $\$ 79.03(S D=40.8, M I N=15, M A X=150)$. Based on this information, I specifically chose anchor values falling outside the range of values

\footnotetext{
${ }^{7}$ I used a similar debriefing technique to that mentioned in study 2 . None of the participants were suspicious of the connection between the two questionnaires.
} 
provided by this pretest group to allow for a stronger test of the rule-priming hypothesis. That is, the choice of implausible anchor values was made to increase the probability that the concept 'expensive' or 'inexpensive' would be activated, resulting in the activation of the rule-relevant concepts of 'heavy' or 'light'.

Immediately after responding to the comparative question, participants were asked to estimate the weight of the textbook (in lbs) they were currently using in their introduction to psychology course, which constituted the main dependent variable. Following this question, participants responded to 25 filler questions, to make the cover story of a questionnaire pretest more convincing. Finally, participants were debriefed, thanked and dismissed.

\section{Results and Discussion}

As the inspection of Figure 2 reveals, the anchoring effect clearly depended on the type of rule participants were primed with, and on the anchor values presented during the comparative question. Specifically, in the 'assimilation' rule condition (expensive - heavy, inexpensive - light), participants gave higher estimates of the weight (in lbs) of their current introduction to psychology textbook $(M=7.7, \mathrm{SE}=$ $1.00)$, after considering a high anchor $(\$ 10,000)$ of the price of a wristwatch, compared to participants who considered a low $(\$ 2)$ anchor $(M=5.19, \mathrm{SE}=1.2)$. This pattern of results was reversed in the 'contrast' rule condition. Specifically, when participants were primed with an 'expensive $=$ light' (inexpensive $=$ heavy) rule, estimates of the textbook weight were lower in the high anchor condition $(M=$ $2.94, \mathrm{SE}=1.2)$, compared to the low anchor condition $(M=5.77, \mathrm{SE}=1.00)$. This pattern of results produced a significant interaction effect in a 2 (high vs. low anchor) 
$\mathrm{X} 2$ (assimilation vs. contrast rule) analysis of variance (ANOVA), $F(1,39)=5.515$, $p<.05$. Neither the main effect of anchor nor the main effect of rule-prime reached significance. Subsequent simple main effects analysis of the anchor effect within each level of the rule-priming factor revealed that neither of the two differences was significant.

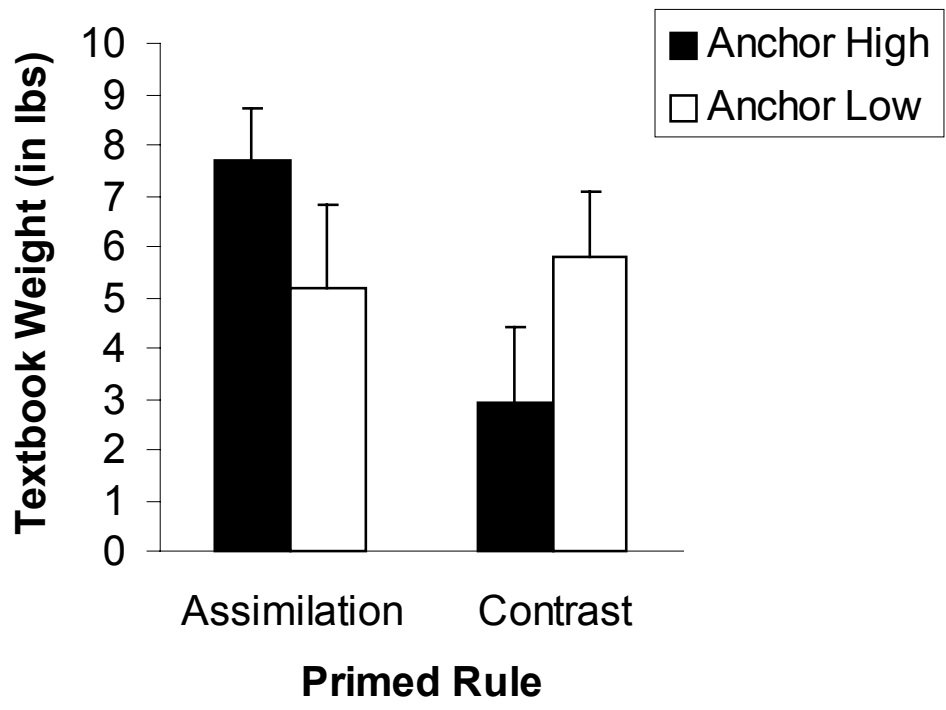

Figure 2. Absolute estimates by rule prime and anchor (study 3).

These results clearly demonstrate that the subjective interpretation of the anchor value itself along a specific semantic dimension (activated via specific ruleprimes) can yield contrast or assimilation effects in anchoring. It is worth noting here that there may exist a virtually limitless number of subjective rules, linking together judgmental dimensions such as "expensive" and "heavy" or "expensive" and "light", depending on a person's subjective experience of the world. Thus, the concept 'expensive' might associatively influence a judgment of weight, if a person thinks of an expensive cell phone (expensive cell phones tend to be lighter and smaller than 
inexpensive ones). This association could yield contrast effects in a variety of weight related estimates, irrespective of specific targets. Similarly, the concept 'expensive' might associatively prime the concept 'heavy' if the person thinks (situationally) of diamond rings. Intriguingly, chronic associations may develop between judgmental dimensions frequently encountered by persons of different occupations, ages, backgrounds, etc., producing either contrast or assimilation effects on estimation tasks. These implications have yet to be fully explored.

Finally, adding to the current activation literature, the above results were obtained despite the fact that targets changed between the comparative and the absolute question and no applicable target-knowledge was accessible, inconsistent with predictions derived from the SA model. Specifically, according to the SA model, judges in the high watch-price condition would have positively tested the hypothesis that the average price of a watch is equal to the $\$ 10,000$ standard, thereby activating standard consistent knowledge that watches are relatively expensive. In the opposite, low anchor condition, participants would have tested the opposite hypothesis, namely that watches are generally inexpensive. According to the SA model, the selectively activated knowledge about watches, however, would not have been relevant to a subsequent estimation of the weight of a textbook, predicting no anchoring effect under these conditions. Similarly, a simple numeric priming perspective (Wilson, Houston, Etling, \& Brekke, 1996) also cannot account for these findings, because it would simply predict assimilation effects in both conditions (e.g., higher estimates after considering a high anchor vs. low anchor), irrespective of rule priming. The results obtained in study 3, thus, highlight the importance of anchor-related concepts 
in producing anchoring effects, while also providing an explanation for assimilation and contrast effects in comparative judgment tasks.

While the first three studies reported herein appear to provide consistent support for an anchor-based semantic priming mechanism, this specific mechanism was recently considered as an alternative explanation to some of the findings reported under the SA model (Mussweiler \& Strack, 2000a). Consider, again, the lexical decision task findings reported by Mussweiler and Strack (2000a), described earlier. Combining a standard anchoring task about average temperatures in Germany with a lexical decision task, these authors found that responses to target words such as hot, warm, summer, beach, swim, sun, sea and oven, cold, snow, ski, winter, sleigh were differentially facilitated, depending on whether participants received a high or a low temperature value during the comparative task. As Mussweiler and Strack acknowledged,

"[...] the obtained results may also be explained by a simple priming mechanism. More specifically, one may argue that processing the given anchor value directly increases the accessibility of words that are associated with the anchor value. [...] As a consequence, anchor-consistent words are recognized faster in a lexical decision task" (Mussweiler and Strack, 2000a, p. 1044).

Apparently, Mussweiler and Strack considered the possibility that a general semantic priming mechanism may be responsible for the production of anchoring effects. However, since "[...] the selective generation of knowledge about the target constitutes the core aspect of [their] model, [...]”" (Mussweiler \& Strack, 2000a, p. 1044), they attempted to rule this possibility out. Thus, in a third study in their series 
of studies, participants in a self-anchoring task were asked to indicate, for example, whether they knew more or less than 30 or 10 countries and their capitals. For half the participants, a subsequent absolute estimate referred to their actual geographical knowledge, whereas for the other half, the absolute question pertained to their father's geographical knowledge. This study revealed an anchoring effect when participants estimated their own knowledge, after comparing their knowledge to a high or low standard, but found no anchoring effect with respect to their father's knowledge. Mussweiler and Strack interpreted this finding as evidence against a general priming mechanism, since general semantics associated with the anchors should have influenced the knowledge of the 'other' target as well.

This finding obtained by Mussweiler and Strack (2000a), on the surface, appears to invalidate the notions put forth in this dissertation. Indeed, from a general priming perspective, general concepts related to knowledgeability, activated during the comparative task, should (logically) have influenced the estimation of the 'other' target as well. How may this inconsistency be reconciled with the studies already described in this project? An explanation may involve a testable assumption concerning the use of information under conditions of changed contextual parameters (e.g., a change in targets from one question to another). Participants in the Mussweiler and Strack (2000a) study, at the time of the absolute judgment, may have made a meta-cognitive judgment, in which they classified the self-knowledge prime as irrelevant, with respect to a judgment about their fathers' knowledge (see implication "4" of this model). Specifically, participants who were asked to estimate their fathers' knowledge, after being asked about their own knowledge, may have 
judged any knowledge, activated in response to considering a 'self' question, as inappropriate to answer the 'other' question. Note that this explanation would be equally applicable to the SA model and the anchor-based semantic priming model. That is, both specific target knowledge, as well as general anchor-based semantics, could potentially have been inhibited by participants in Mussweiler and Strack's (2000a) study, resulting in the absence of an anchoring effect when the target changed from self to other.

The ability to inhibit knowledge or notice an 'inappropriate' change in judgmental targets should be dependent one's level of cognitive capacity, a parameter known to affect the processing of a large variety of judgments (e.g., see Chun, Spiegel, \& Kruglanski, 2002). Thus, reductions in cognitive capacity at the time of the absolute judgment may decrease a judge's ability to make meta-cognitive usability judgments, or notice the change in targets as an 'alert' signal to inhibit the prime (see also Martin \& Achee's, 1992 notion of 'resetting' in judgmental tasks). Following this logic, under cognitive load, judges may actually assimilate the 'other' judgment to the general prime, activated during a 'self' judgment. Under conditions of normal capacity, Mussweiler and Strack's findings (2000a) should replicate, because judges have the ability to notice the change in targets and meta-cognitively inhibit irrelevant information. ${ }^{8}$ Study 5 was designed to address these questions, empirically.

\footnotetext{
${ }^{8}$ It should be noted that a change in judgmental targets between comparative and absolute questions may not always be perceived as a warning signal to inhibit knowledge that was activated during the comparative question. For example, participants may not think that consecutive questions about the age of a person and the price of a watch are intentionally linked within a 'general knowledge' experiment. However, being asked to compare one's own geographical knowledge to a standard before being asked to estimate one's father's geographical knowledge are likely to be perceived as two intentionally related tasks. Thus, judges may inhibit the use of information more often in situations
} 


\section{$\underline{\text { Study } 4}$}

\section{Method}

Participants. 75 (male and female) students at the University of Maryland participated in exchange for course credit in an introductory psychology course.

Procedure and Materials. Participants were, again, recruited under the pretext of a study designed to pretest the construction of a general knowledge questionnaire. After receiving seven identical practice question-pairs, all of the participants responded to the critical anchoring questions. The content of the critical comparative questions was identical to the one used by Mussweiler and Strack (2000a). Only the anchor values chosen for this study were changed. Specifically, a group of participants serving as a calibration group $(\mathrm{N}=26)$ gave responses to only the absolute questions used in this study. The anchor values that were chosen constituted values falling roughly one standard deviation above and below the mean of the estimates provided by the calibration group. Thus, the anchor values were equally extreme as those chosen by Mussweiler \& Strack (2000a), but were calibrated to reflect the knowledge of University of Maryland students. All comparative questions pertained to the self as the judgmental target. For example, in the first question, participants were asked whether they would be able to list more or less than either 4 or 26 countries and their capitals and whether they would be able to list more or less than 2 or 14 US rivers.

when the same question is asked twice (e.g., pertaining to geographical knowledge), but about different targets. 
Depending on condition, participants were then informed that they would have to respond to the next general knowledge question, while trying to remember an 8-letter nonsense syllable (DBHNRKTY). They were told that people commonly have to remember a large amount of information while simultaneously working on a task and that trying to remember the 8-letter nonsense syllable would more accurately reflect conditions in real life. The other half was simply asked to give an exact estimate of how many countries/capitals and rivers they knew or provide an estimate with respect to their father's knowledge. It should be noted that in Mussweiler and Strack's study, the absolute question was replaced with a self vs. other free description task. Specifically, participants were asked to describe themselves or their father for a few minutes and that the foremost interest of the researchers was in their or their father's factual knowledge. For the sake of simplicity (and generalizability to the anchoring domain), the study conducted in this dissertation included a standard absolute question, which asked participants to provide a numerical estimates of how many rivers and countries/capitals they knew.

Thus, this study was based on a 2 (anchor high vs. low) X 2 (cognitive load vs. no load) X 2 (self vs. other as target of absolute judgment) design.

\section{Results and Discussion}

An initial analysis of the data revealed no significant difference of question content (countries/capitals vs. rivers) on the pattern of results obtained in this study. As a consequence, an overall 2 (anchor high vs. low) X 2 (load vs. no load) X 2 (self vs. other as target of absolute judgment) analysis of variance (ANOVA) was performed on a combined index of geographical knowledge (combined countries + 
capitals and US rivers). The analysis revealed only a significant main effect of anchor, $F(1,66)=18.722, p<.001$ and a main effect of target, $F(1,66)=10.063, p$ $<.01$. As the inspection of Figure 3 reveals, participants consistently provided higher estimates of geographical knowledge in the high anchor condition, compared to the low anchor condition. Furthermore, the main effect of target indicated that participants gave higher average estimates of their father's knowledge $(M=27.31, \mathrm{SE}$ $=2.1)$ compared to their own knowledge $(M=17.74, \mathrm{SE}=2.2)$.

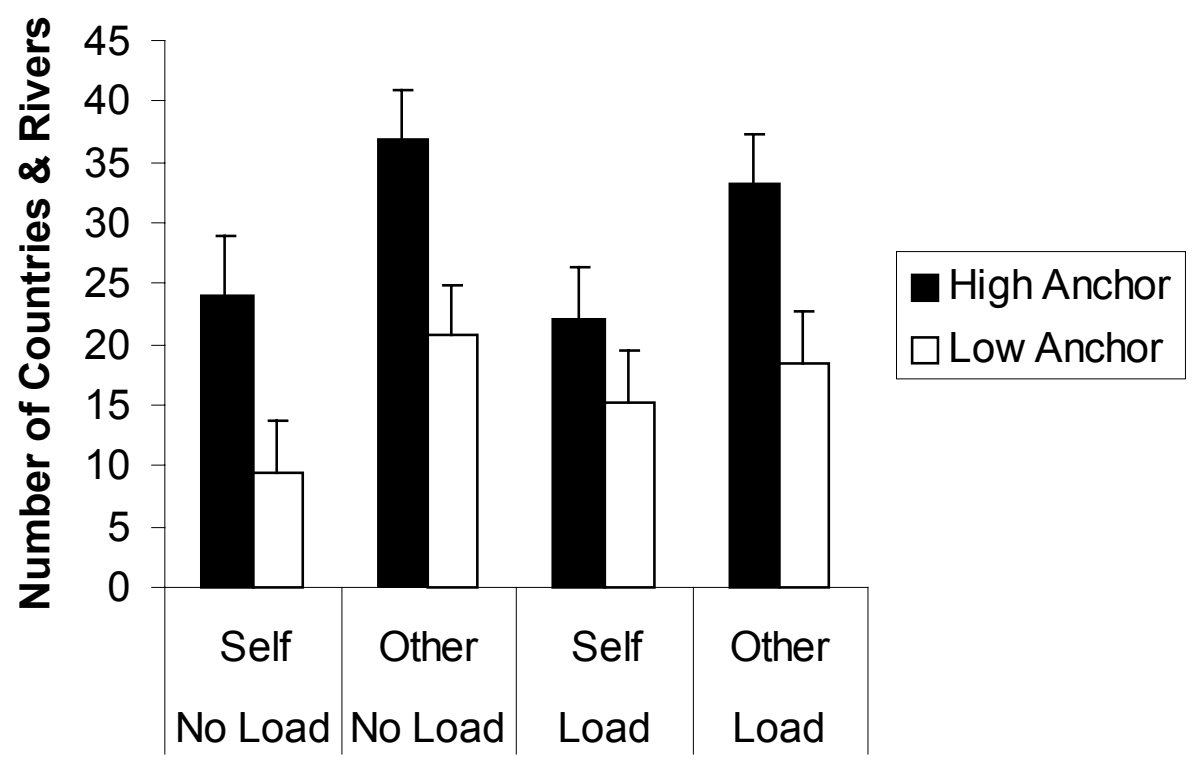

Figure 3. Absolute estimates of geographic knowledge (countries + capitals, and US rivers combined) by anchor, target and load (study 4).

The anchoring effect occurred irrespective of whether the absolute judgment pertained to the self or to another person (participants' fathers) and irrespective of load. Specifically, I had expected participants in the four 'no load' cells to only be influenced by the anchor values when the absolute question pertained to themselves and to inhibit the anchor-based prime of knowledgeability when the question 
pertained to their father's knowledge. Thus, I had expected to find an interaction between target and anchor in the 'no load' condition, but not in the load condition. The results of this study failed to reflect this particular pattern.

The results obtained in this study are readily interpretable in terms of the anchor-based priming mechanism outlined in this dissertation, which predicts that general semantic notions mediate anchoring effects across a range of targets (e.g., self and other judgments). However, due to the assumed operation of meta-cognitive correction processes, I also expected an absence of an anchoring effect when the target of the judgment was perceived to have changed (from self to other, involving the same question), given participants' sufficient capacity to notice the change (i.e., in the 'no load' condition). Thus, in the 'no load' condition, this study fails to replicate the findings obtained by Mussweiler and Strack (2000a, study 3), who showed that free descriptions of their participants' own knowledge were influenced by the anchor manipulation, whereas descriptions of the participants' father's knowledge were not.

One obvious explanation for the difference in results obtained between the two studies involves the variation in the measures of the dependent variable. Whereas Mussweiler and Strack (2000a) engaged their participants in a free description task, involving general knowledge, participants in the present study were simply asked to provide a numerical estimate of general knowledge (consistent with the typical anchoring paradigm). It seems plausible that participants, who were asked to provide a 'snapshot' numerical estimate of their father's geographical knowledge, may have failed to inhibit the anchor-based prime concerning their own geographical knowledge, because these estimates were likely to have been generated quickly and 
without much thought, even in the 'no load' condition. When participants, on the other hand, were induced to write about their father's general knowledge, they may have been more thoughtful in the way they chose to characterize their fathers, hence inhibiting the self-knowledge prime. These possibilities point, perhaps, to a difference in the extent to which anchor-based primes exert their influence under conditions of changed targets, depending on the nature of the judgmental task at hand. Specifically, when the judgmental task involves only a 'quick and dirty' numerical estimate, judges may provide it without paying much attention to the fact that knowledge, primed prior to the estimation task, may be irrelevant (e.g., under conditions of certain changes in context). On the other hand, when the judgmental task is inherently more effortful and lengthy (e.g., a free description task), anchor-based primes may be more readily inhibited under conditions of changed targets, because judges are likely to be more thoughtfully engaged in those types of tasks, given sufficient levels of motivation or cognitive capacity. A follow-up study, using Mussweiler and Strack's (2000a, study 3) free description task, along with the load manipulation, is currently being conducted. Provided the above logic is correct, this follow-up study should replicate Mussweiler and Strack's (2000a) findings in the 'no load' conditions and replicate the pattern of results obtained in the present study under load conditions (e.g., anchoring effects irrespective of target). If these results were obtained, they would provide a general explanation for the typical robustness of the anchoring effect, using numerical estimates as dependent variables, and for the absence of the effect under conditions of changed targets, involving measures of the dependent variable, requiring more thought. 
Regardless of the outcome of this follow-up study, the apparent robustness of the anchoring effect, as evidenced in this study by a) an effect across different judgmental targets (self vs. other) and b) an effect that resulted independent of load, appears striking. Furthermore, this generality of the anchoring effect appears to strongly afford the conclusion that general semantic notions related to the anchor values themselves may be at work. However, although notions of positive hypothesis testing and selective accessibility (Strack \& Mussweiler, 1997) are unable to account for these results, one could argue that the basic (numeric) anchoring mechanism proposed by Wilson et al. (1996) provides a ready explanation for the patterns observed in study 4. Simply put, a short-term memory representation of the anchor value itself could have influenced the estimates of geographic knowledge in this study, accounting both for the effect across targets (self vs. other) and the load independence of the effect.

In order to provide more unequivocal support for the anchor-based semantic priming explanation of anchoring effects under conditions of changed judgmental targets, I chose to vary the typical anchoring paradigm in a fifth study. Consider again the nature of the comparative question in the typical anchoring paradigm, in which judges consider either a high or a low anchor value as a comparison standard. While the anchor values vary between subjects, the target of the comparative question is generally not varied. As a result, judges may be primed with different semantic notions, because the anchor values have different meanings, but in connection to the same target. For example, after being asked whether former President Clinton is older or younger than 98 years, one will likely be primed with words related to the semantic 
category 'old'. A lower standard of his age (e.g., 18 years) would prime the opposite concept 'young'. Within the context of this specific paradigm, however, it is relatively difficult to tease apart whether judges are indeed influenced by general semantic notions related to the anchor values, or, alternatively, by the numerical values themselves, in arriving at an absolute estimate (Wilson et al., 1996). This is especially so when the target of the absolute judgment is different from the target of the comparative judgment (e.g., see results of study 4).

Perhaps the most direct test of the anchor-based semantic priming explanation, thus, would involve a variation of the typical paradigm, in which the numerical anchor values are held constant in a between-subjects design (e.g., the design employs identical numerical anchor values), but the target of the comparison is varied in such a way that the same number (or anchor value) primes different semantic concepts, depending on the nature of the target ${ }^{9}$. An obtained anchoring effect, using this design, could not be accounted for by a pure numeric priming explanation of anchoring, because the numerical values are identical between conditions.

To test these notions, I employed a comparative question involving the age of two targets, using the same numerical anchor value as a comparison standard. Specifically, depending on condition, participants either received a comparative question involving a well-known 'older' target person or a well-known 'younger' target person. Participants in both conditions received the same anchor value, which

\footnotetext{
${ }^{9}$ While prior research has shown (Mussweiler \& Strack, 1999) that the same number (e.g., 3000) can vary in perceived plausibility as a function of different target categorizations (e.g., height of a mountain, age of a person), this research still employed the traditional version of the comparative question, varying the anchor values (high vs. low) in addition to the categorization of the target (e.g., mountains vs. people).
} 
fell roughly in the middle of a range of values considered plausible for human age. Thus, in one condition, in which the 'young' target was compared to the anchor, I predicted the concept 'old' to be primed, because the anchor value constituted a high standard of the target person's age. Following the same logic, when the 'old' target person was compared to the identical anchor value, the concept 'young' was predicted to be primed (again, because the anchor value would be considered a low standard of the person's age). Following the comparative judgment phase, all participants estimated the age of a third, unrelated target.

\section{$\underline{\text { Study } 5}$}

\section{Method}

Participants and Design. 24 (male and female) students at the University of Maryland participated in exchange for course credit in an introductory psychology course. They were randomly assigned to the two cells of a modified anchoring design.

Procedure and Materials. As in the previous four studies, participants participated under the pretext of a study, designed to develop different wordings for general knowledge questions. After receiving general instructions similar to those used in the first four studies, all participants responded to 8 filler questions (absolute estimation tasks regarding a number of different targets). Afterwards, the critical independent variable was presented. Depending on condition, participants were asked to indicate whether pop singer Britney Spears was older or younger than 45 years, or whether Hollywood actor Clint Eastwood was older or younger than 45 years. These targets were chosen to reflect well-known exemplars of the categories 'old' and 
'young'. Pre-testing $(\mathrm{N}=26)$ prior to this study had revealed that students generally knew a) who Britney Spears and Clint Eastwood were and b) that Britney Spears was relatively young and Clint Eastwood relatively old. The anchor value was chosen to reflect the midpoint of a range of plausible values for human age, which therefore constituted a high standard for Spears age and a low standard for Eastwood's age. Following this manipulation, all participants were asked to estimate the ideal age of a bottle of French red wine, before it should be opened. This question constituted the main dependent variable. Finally, all participants responded to a number of filler questions, before they were debriefed, thanked and dismissed.

\section{Results and Discussion}

The anchoring effect was replicated. The results of a one-way analysis of variance (ANOVA) showed that participants gave higher estimates of the ideal age of a bottle of French red wine in the Britney Spears comparison condition $(M=27.68$, $S D=15.16, N=13)$, relative to the Clint Eastwood comparison condition $(M=14.88$, $S D=13.43, N=11), F(1,22)=4.709, p<.05$.

These results appear to strongly afford the conclusion that absolute estimates in anchoring tasks may be based on general semantic notions, directly related to the anchor value itself. Specifically, when the anchor value (45 years) was presented as a comparison standard for the age of a young person (Britney Spears), participants gave higher estimates of the age of an unrelated target (French Wine), presumably because the concept 'old' became activated after processing the comparative question. That is, because of the generality of the concept 'old' (as opposed to target-specific knowledge about Spears or Eastwood), this semantic category appeared to have 
influenced a subsequent estimate of the age of a bottle of wine, despite the fact that the concept was activated in a different context. Similarly, when the same anchor value served as a standard of comparison for an older person (Clint Eastwood), participants appeared to have been primed with the concept 'young' (45 years constituted a low standard for the his age), resulting in lower estimates of the age of a bottle of wine. Because the anchor values themselves were specifically not varied in this study, an interpretation of these results based on a numerical priming perspective (Wilson et al., 1996) can be ruled out.

With pure numerical priming ruled out, could Strack and Mussweiler's (1997) SA model account for this finding? The answer appears to be negative. According to their model, participants in the Britney Spears condition presumably tested the hypothesis that Britney Spears was 45 years old, thereby activating standardconsistent knowledge about her (e.g., specific knowledge that she is relatively old). Similarly, participants testing the hypothesis that Clint Eastwood was relatively young (e.g., 45 years of age) presumably activated knowledge about the actor that would be consistent with the notion that he is young. According to the SA model's 'representativeness' constraint of activated knowledge, no anchoring effect (or a contrast effect indicating over correction) should have been obtained, because the target of the absolute judgment (involving French red wine) was not representative of either target of the comparative judgment (Britney Spears, or Clint Eastwood). Thus, the present results also rule out the SA model as an explanatory framework for this particular effect. 
One weakness of the current design is that it does not rule out a third alternative interpretation of the obtained pattern of results, not predicted by any of the current models of anchoring. Specifically, while thinking about Britney Spears, participants may have been primed with the concept 'young', after providing an answer to the comparative question. Because every participant in this study indicated that Britney Spears was younger than 45 years, they may have been primed with the concept young, rather than the concept old, after processing the comparative question. Similarly, because every participant indicated that Clint Eastwood was older than 45 years, participants in this condition may have been primed with the concept old, rather than the anchor-based concept of young. Within the context of this alternative priming mechanism, the obtained results may have been due to a contrast effect. That is, the higher estimates of age of the bottle of wine in the Britney Spears condition (compared to the Clint Eastwood condition) may have been the result of an over correction process: Participants may have perceived a change in targets between the comparative question involving the age of a celebrity and the age of a bottle of wine, and corrected for the potential influence of the alternative 'young' prime in the Spears condition and the 'old' prime in the Eastwood condition on their estimates of wine age.

It should be noted here that this explanation appears to be an unlikely candidate to explain these results, because the design of this study was essentially analogous to a study involving implausible anchor values. More specifically, the value 45 is clearly an implausible candidate for both Britney Spears and Clint Eastwood's age and similar in that respect to any other anchoring study involving 
implausible anchors (e.g., “was Mahatma Gandhi older or younger than 140 years?”). In most implausible anchoring paradigms, the actual answer to the comparative question does not appear to influence the effect at all. In fact, as previously mentioned, most implausible anchoring studies yield stronger anchoring effects, compared to plausible anchoring studies. Thus, despite the fact that participants generally know that the answer to the comparative question is lower or higher than an implausibly high or low anchor value, the fact that absolute estimates are almost always consistent with the implication of the anchors, does not appear to indicate that the actual answer to the comparative question primes participants with values opposite to those implied by the anchor value (e.g., an answer "younger than 140 years of age" does not appear to prime "young").

Be that as it may, a final, sixth study was conducted to rule out this alternative explanation, replicating and extending the results obtained in study 5. Using the combined anchoring and lexical decision task paradigm employed in study 1, study 6 sought out to demonstrate an increased level of lexical activation of the concept 'old' in the Britney Spears condition, relative to the level of activation of the concept 'young' in that condition. Similarly, I predicted a higher level of lexical activation of the concept 'young' in the Clint Eastwood condition, relative to the concept 'old'. To add to the generality of the modified anchoring paradigm introduced in study 5 , the dependent variable in study 6 pertained to the age of a person, as opposed to the age of an object. 


\section{Study 6}

\section{Method}

Participants. 26 (male and female) students at the University of Maryland participated in exchange for course credit in an introductory psychology course.

Procedure and Materials. The procedure and materials used in this study were identical to those used in study 5 with the following exceptions. All materials were presented on IBM-compatible workstations using the MediaLab ${ }^{\mathrm{TM}}$ software program, instead of questionnaires. Following the general instruction phase and the filler questions, participants received either the Britney Spears or Clint Eastwood comparison question, before being told that the next section would test their general knowledge in a novel way. Participants then received instructions on how to respond to the lexical decision task. The stimulus materials (e.g., letter strings), and procedures used in the lexical decision task were identical to those used in study 1 . As in study 1, the task included words related to the semantic categories 'old' and 'young', as well as unrelated words and nonwords (see table 1 for letter strings used in this task). After participants completed the lexical decision task, they were presented with the dependent variable. Specifically, they were asked to estimate the age of current US senator Robert Bennett (R-UT). Finally, participants responded to 25 filler questions before being debriefed, thanked and dismissed from the experiment. 


\section{Results and Discussion}

Absolute estimates. The results of this study replicated the anchoring effect obtained in study 5. Specifically, participants gave higher estimates of senator Bennett's age in the Spears condition $(M=58.23, S D=6.77, N=13)$, compared to the Eastwood condition $(M=51.38, S D=6.76, N=13)$. A one-way analysis of variance (ANOVA) performed on these estimates yielded a statistically significant effect, $F(1,24)=6.651, p<.05$.

Response latencies. As in study 1, all individual response latencies were first transformed, using a natural logarithmic transformation. Subsequently, individual baseline indexes of responding were calculated by averaging individual responses to the noncritical words and the nonwords. For each participant, this index was subtracted from the average responses to the 'old' and 'young' words, respectively. Thus, the index used in the subsequent analysis constituted participants' individual deviations from their baseline speed of responding. Negative values represent responses that are faster than this baseline; positive values represent responses that are slower.

As the inspection of table 4 reveals, participants' responses to the old and young words depended on whether they compared Britney Spears' age to the 45-year anchor or Clint Eastwood's age to that same anchor. Specifically, participants were faster at recognizing 'old' related words in the Spears condition, compared to the Eastwood condition. Conversely, participants in the Eastwood condition were faster at recognizing 'young' related words, compared to the 'old' related words. This pattern produced a significant effect in a 2 (Spears v. Eastwood as comparison target) 
$\mathrm{X} 2$ (old vs. young words) mixed model analysis of variance (ANOVA), $F(1,24)=$

$12.184, p<.01$. None of the other main effects reached significance.

Table 4

Response latencies for lexical decisions for old and young words by anchor (Study6).

Target

\begin{tabular}{|c|c|c|c|c|}
\hline \multirow[b]{2}{*}{ Anchor } & \multicolumn{2}{|c|}{ Old Words } & \multicolumn{2}{|c|}{ Young Words } \\
\hline & M & SD & M & $\mathrm{SD}$ \\
\hline $\begin{array}{c}\text { High } \\
\text { (Spears) }\end{array}$ & -.174 & .064 & -.129 & .067 \\
\hline $\begin{array}{c}\text { Low } \\
\text { (Eastwood) }\end{array}$ & -.045 & .075 & -.158 & .096 \\
\hline
\end{tabular}

Note. Deviations from the baseline speed of responding to neutral and nonwords (in $\log \mathrm{ms}$ ) are reported. Lower values indicate faster responses.

The results obtained in this study further demonstrate the profound influence of anchor-based semantics on judgmental anchoring and provide additional evidence for the operation of this mechanism. Specifically, analyses of response latencies to anchor-based semantics in a lexical decision task indicated that participants were clearly primed with the anchor-based concept 'old' in the Britney Spears condition and, to a somewhat lesser degree, with the anchor-based concept 'young' in the Clint Eastwood condition. Thus, the pattern of response latencies obtained in this study provides strong evidence against the alternative explanation of the results obtained in study 5 and 6 . In particular, because the lexical activation pattern is consistent with the anchoring effects obtained in both studies, it seems unlikely that participants were primed with the concept 'young' in response to their answer to the Britney Spears 
comparison question, and with the concept 'old' after processing the Clint Eastwood comparison question.

In sum, the anchoring effects obtained in studies 5 and 6 , in combination with the lexical decision results obtained in study 6 , strongly afford the conclusion that semantic notions directly related to the anchor values are sufficient in producing anchoring effects. 


\section{General Discussion}

Individuals are often faced with significant amounts of uncertainty when it comes to a wide array of judgmental tasks and decisions in their daily lives. Persons might, for example, wonder how much money they should offer for a piece of real estate, whether they are being paid enough at their jobs, whether they should go to Hawaii or Barbados for vacation, or how they are measuring up to a faceless competition on the current job market, and so forth. These and many other questions are often difficult to answer from within an informational vacuum. Having access to at least some kind of comparison standard, within a given judgmental context, typically proves to be of significant informational value. For example, to know the average amount of money that was paid for a piece of real estate in the past provides information as to how much one should currently offer for the object. To know that a colleague gets paid a certain amount of money to do a type of work similar to one's own professional responsibilities, helps in determining whether one should ask for a raise or not. Or, to know that a position had over 100 applicants, but another had only 20, would aid in determining the respective desirability or competitiveness of the two positions, and so forth. Social psychologists have long recognized the importance and usefulness of comparison standards within the domain of social judgment (e.g., Festinger, 1954), and so, the fact that standards of comparison are frequently sought out by individuals as important sources of information is hardly surprising, and appears logically and strategically sound. 
What has puzzled psychologists for three decades now, based on the groundbreaking research by Tversky and Kahneman (1974), is the apparently irrational tendency for individuals to be influenced by completely uninformative comparison standards, in reaching a judgment about a target. Studied primarily in the domain of numerical comparisons and judgments, individuals have, time and again, been shown to base their estimations, related to a host of targets, on (ostensibly) randomly selected, and hence uninformative, standards of comparison. While psychologists have spent much of the last 30 years demonstrating this type of anchoring effect, in a variety of judgment and decision-making domains, a recent influx of theoretical contributions to the social judgment literature has begun to shed promising light on the psychological processes that may be involved in judgmental anchoring effects.

The key purpose of the present dissertation project was to integrate the major existing theoretical notions of anchoring into a single mechanism that can account for a broad range of anchoring effects. I essentially proposed that a comparison of a given target with a numerical anchor activates semantic notions that are directly related to the anchor value. A subsequent absolute estimate of the target feature was hypothesized to be based on the semantic notions related to the anchor values, and not necessarily on the anchor values themselves (Wilson et al., 1996), or on target specific knowledge that was activated in response to a positive hypothesis test (Strack \& Mussweiler, 1997).

Study 1 supported the hypothesis that anchor-based semantics may be responsible for producing anchoring effects by showing that differentially extreme 
anchor values appear to be associated with different levels of activation of anchorrelated words. Specifically, using response latency data, the results of study one showed that comparisons with implausible anchors result in an increased activation of words related to the anchor values, compared to comparisons with plausible anchors. These differences mapped on to a larger anchoring effect obtained in the implausible vs. plausible condition, providing an explanation for the larger effects of implausible anchors. In study 2, participants were primed directly with anchor-related words, which resulted in a 'mimicked' anchoring effect. Thus, although no specific targetanchor comparison was made, participants assimilated their numerical estimates of a target (the average length of a whale) to a set of primed constructs that presumably reflected notions related to high and low anchors of the length/size dimension, used in this study. Study 3 showed that associatively activated concepts, primed via a conditional rule, produce anchoring effects when the target and dimension of the judgment change between the comparative and absolute questions. The pattern of results obtained in study 3 also provided evidence for the prediction that associative knowledge activation processes may be responsible for producing contrast effects in anchoring. Study 4 failed to support the prediction that a target change between a comparative task and related absolute estimate, involving the same judgmental question, inhibits anchoring. Nonetheless, this study was strongly supportive of a general semantic priming mechanism, evidenced by the robustness of the anchoring effect, which was obtained across different targets (self v. other) and independent of cognitive load. Study 5 varied the typical anchoring paradigm by holding the numerical anchor constant and varying the target of the comparative question, 
producing a purely semantic anchoring effect. Finally, study 6 replicated and extended the findings obtained in study 5, providing additional evidence (from a lexical decision task) that anchor-based semantic notions mediate anchoring effects.

\section{Relation to the SA model}

The theory of anchor-based semantic priming, proposed in this work, is directly based on insights gathered from the selective accessibility work of Strack and Mussweiler, and in many ways constitutes a simplification of the basic SA process. My perspective primarily differs from existing notions of selective accessibility in the kinds of semantics that are assumed to mediate anchoring effects and in the role of target-knowledge in anchoring. Specifically, I assume that a positive hypothesis testing mechanism that increases the accessibility of selective target knowledge may not constitute a necessary mechanism for anchoring effects to obtain. In making an ambiguous numerical estimate of a target quantity, judges may simply base their estimations on a general semantic concept or category that is primed during the initial target-standard comparison. For example, when comparing Mother Theresa's age at her death to a high anchor value (e.g., 89) a general concept such as "old" may be primed, which subsequently forms the basis of the absolute estimate. Similarly, comparing her age to a low anchor would prime the opposite concept ("young"). A large number of studies in the social-cognitive literature support the contention that target evaluations may be influenced by the accessibility of general concepts. In what is probably the most well-known study of this kind, Higgins, Rholes, and Jones (1977) demonstrated that an ambiguous behavior of a target person "Donald" was interpreted in terms of two different traits (e.g., adventurous or reckless), depending 
on which one of the two traits was primed in an earlier part of the study. An assumption that judges positively test the hypothesis that Donald is either adventurous or reckless is not needed to explain these kinds of knowledge accessibility effects. Rather, judges may simply perceive the subjective relevance of the activated trait concept (e.g., reckless) for the interpretation of the ambiguous target behavior.

This simplification of the SA process allows for an integrated explanation of implausible and plausible anchoring effects in terms of the same, simple priming mechanism. An 'adjustment' process, therefore, is not postulated in the present model, because implausible anchoring effects can be explained by an increase in accessibility of anchor-related words, after considering an implausible vs. plausible anchor value.

\section{Correction processes}

Consistent with the social cognitive literature on priming, or knowledge activation in general, is the notion that anchor-based semantics may sometimes be perceived as irrelevant for a subsequent judgment (e.g., Higgins, 1996). Specifically, under certain circumstances (e.g., same question, but different targets of the absolute and comparative judgment), judges may perceive the general semantic notions, activated during the comparative judgment, to be irrelevant for a subsequent absolute judgment. Study 4 conducted as part of this dissertation failed to support this notion, in a condition in which participants were first anchored on their own geographical knowledge before being asked to estimate another person's level of geographical knowledge. Although the change in targets between the comparative and absolute 
judgment tasks should have been perceived as illegitimate, in terms of using the selfknowledge prime, participants nonetheless anchored the other target's geographical knowledge on semantic notions that were activated during a self-comparison task.

This finding constitutes an inconsistency to the findings obtained by Strack and Mussweiler's (2000a) and to their conceptualization of the anchoring effect. Specifically, these authors assume that knowledge, activated via a positive hypothesis test, will not influence absolute judgments, unless judges perceived the knowledge to be relevant (i.e., applicable and representative) to a subsequent judgment. Because, according to the SA model, anchoring effects are mediated by target-specific knowledge, the anchoring effect was described to be limited to the traditional paradigm, in which the target of the comparative question is identical to the target of the absolute question (Mussweiler \& Strack, 2000b). The present theory differs from the SA model in this regard, because the knowledge activated in response to processing a comparative question is hypothesized to be sufficiently general in nature to allow for cross-target effects as well. That is, although the activation of anchorbased semantics is hypothesized to depend on the categorization of the target and the anchor value at the time of the comparative judgment, once general semantic notions are activated, they hold the potential to influence a number of subsequent judgments. These types of cross-target effects, however, may be dependent on the nature of the absolute estimation task. Specifically, the differences in results between study 4 in this series and Mussweiler and Strack's (2000a) third study suggest that quick numerical estimates appear to be unaffected by meta-cognitive considerations that potentially inhibit anchoring effects (study 4 of the present project). However, judges 
may still inhibit objectively irrelevant knowledge on more effortful tasks (such as a free response description related to the judgmental dimension) as was demonstrated by Mussweiler and Strack (2000a). Whether or not there truly are replicable differences in meta-cognitive correction processes, as a function of the type of estimation task involved (e.g., short vs. lengthy) could be fruitfully examined in future research.

\section{Relation to basic anchoring}

One of the goals of the present research was to empirically distinguish the presently proposed theory of anchoring from the basic anchoring notions put forth by Wilson et al. (1996), who argued that judges may exhibit anchoring in 'changed target' paradigms, due to the influence of a short-term memory representation of the anchor value. While Strack and Mussweiler's (1997) model does not account for (assimilative) anchoring effects when the target of the judgment changes between the comparative and absolute judgment task, both Wilson et al.'s (1996) numeric priming as well as the present anchor-based semantic priming theory predict cross-target effects, based on the explication of more general, mediating psychological mechanisms. For example, in one study described by Wilson et al. (1996), in which participants low in geographic knowledge compared the number of countries in the United Nations to an anchor value (1930) or the number of physicians in the phonebook to that same anchor value, showed anchoring effects with comparable effect sizes (compared to a no anchor control condition), when all participants estimated the number of UN nations. While Wilson et al. (1996) argued that participants in both conditions merely remembered the anchor values (e.g., 1930) I 
would argue that the number (e.g., 1930) used in both comparison tasks (UN nations and physicians) activated the notions "a lot", or "much" (relative to the control condition in their study). These semantic notions later influenced the absolute question involving the number of countries in the UN.

A number of studies in this dissertation appear to favor the latter explanation over Wilson et al.’s (1996) model of anchoring. For example, general concepts related to the dimension of length influenced estimations of the length of a whale, in study 2. Since this study did not include any numerical anchor values, Wilson et al.' model cannot account for this effect. Similarly, in studies 5 and 6 , in which the numerical value of the anchor was held constant between subjects, significant anchoring effects were obtained by varying the target of the judgment, thereby imbuing the anchor values with different meanings. Again, Wilson et al.'s numeric priming model is unable to account for this effect, because the numerical anchors were held constant.

It should be noted here that some studies in the Wilson et al. (1996) series included a paradigm, in which participants did not make any explicit comparisons with the anchor value, but merely were engaged in a task that increased the accessibility of differentially valued numbers in short-term memory. For example, participants in one study were asked to copy a series of numbers under the guise of a 'graphology' experiment, thereby increasing the accessibility of those numbers. As Wilson et al. (1996) showed, anchoring effects were obtained on a subsequent estimation task as a function of the value of the numbers used in the graphology study. A subsequent attempt to replicate this effect, using a different set of numbers 
failed (Brewer \& Chapman, 2002). Because these types of no-comparison numeric effects are difficult to replicate, using a set of numbers that differs from those used by Wilson et al. (1996), Brewer and Chapman concluded that the basic anchoring effect in its purest form (not involving comparisons) is rather fragile. Thus, while reliable anchoring effects are likely to be obtained by priming semantic associates of the anchor values (without explicit comparisons, see studies 2, 5 and 6), a similar procedure involving merely numerical values appears to be difficult to replicate. From the point of view of the present theory, this replication failure of pure numeric effects does not seem surprising, because numerical values by themselves do not appear to be meaningful without proper contextualization, and thus, appear less relevant to a judgment than an anchor-related semantic concept (see Pierro, Mannetti, Kruglanski, \& Sleeth-Keppler, 2004). On the other hand, once numerical values are contextualized, they may prime powerful semantic associates, that subsequently influence a variety of judgments.

Finally, the present work introduced an associative knowledge activation process that is able to account for contrast effects as well as assimilation effects in anchoring. Thus, in addition to contrast effects that are obtained as a function of meta-cognitive correction processes, judgmental contrasting away from the anchor value may be fruitfully predicted in terms of a rule-based mechanism, in which judges associatively activate relevant semantic concepts, after considering a given anchor value. In these terms, study 3 in this series showed that priming an associative rule before having judges compare the average price of a watch to a high or low anchor value, influenced subsequent estimations of the weight of a book. The direction of the 
weight estimate, relative to the anchor value, depended on the nature of the rule that was primed before the anchoring task.

Interestingly, the process of anchor-based (associative) semantic activation provides a framework for the explanation of a number of studies that show a 'spreading' influence of anchor values onto a variety of judgments. For example, in a study by Chapman and Bornstein (1996), which examined the effects of anchoring and adjustment in a legal setting (using mock jurors), found that anchoring participants on a high or low compensations amount for a given personal injury claim not only affected the final compensation amount awarded by the jurors but also affected estimates of the probability that the defendant actually caused the plaintiff harm. This finding could be interpreted as reflecting the operation of a rule-based mechanism. Thus, participants in this study may have adhered to a subjective rule that 'if a high compensation amount, then the crime is severe' (relative to a low compensation amount). The high probability estimate that the defendant caused the plaintiff harm may have been due the activation of the concept 'severe crime', in response to receiving a high compensation request from the trial lawyer.

\section{Future research directions}

The presently proposed anchoring mechanism yields the potential for a number of future research endeavors. For example, while the present set of studies provides evidence for the influence of anchor-related words on anchoring, the specificity of the influence of these words on subsequent judgments could be investigated further. For example, it is unclear, on the basis of these studies, whether

considering an anchor value within a given context primes dimension-specific notions 
(e.g., 'old', 'expensive', 'long', 'wide') or more dimension independent semantic concepts such as ('a lot', 'a little', 'much', 'few', etc.). Thus, in the latter case, anchors, considered across a variety of judgmental dimensions, could be represented in terms of general semantic notions such as 'a lot', or 'a little'. If that were the case, one could present participants with a virtually unlimited range of targets, unrelated to the comparative question, and an anchoring effect would occur. If, however, the semantic notions are specifically tied to the judgmental dimensions under consideration during the comparative judgment task, a subsequent target estimated should only be affected by the anchor value if the estimation is along a similar judgmental dimension, to that considered during the comparative task. These and many other questions, for example, involving the degree to which considerations of anchor values change an individual's representation of the target in memory, have yet to be examined. 


\section{Appendix}

\section{Personal Knowledge Questionnaire}

Gender:

Please complete the questions below. Notice that the response scale varies between

Light vs. Heavy and Expensive vs. Inexpensive. With regard to each question, you may use the most appropriate scale to circle your answer (either Light/Heavy or Expensive/Inexpensive).

1. What is the weight of a feather?

$\begin{array}{lllllll}\text { Inexpensive } & & & & & \text { Expensive } \\ 1 & 2 & 3 & 4 & 5 & 6 & 7 \\ \text { Light } & & & & & & \text { Heavy }\end{array}$

2. What is the price of a 2-karat diamond?

$\begin{array}{lllllll}\text { Inexpensive } & & & & & \text { Expensive } \\ 1 & 2 & 3 & 4 & 5 & 6 & 7 \\ \text { Light } & & & & & \text { Heavy }\end{array}$

3. What is the weight of a swordfish?

$\begin{array}{lllllll}\text { Inexpensive } & & & & & \text { Expensive } \\ 1 & 2 & 3 & 4 & 5 & 6 & 7 \\ \text { Light } & & & & & & \text { Heavy }\end{array}$

4. What is the price of a gallon of gasoline?

$\begin{array}{lllllll}\text { Inexpensive } & & & & & \text { Expensive } \\ 1 & 2 & 3 & 4 & 5 & 6 & 7 \\ \text { Light } & & & & & & \text { Heavy }\end{array}$

5. What is the price of a meal at McDonalds?

$\begin{array}{lllllll}\text { Inexpensive } & & & & & \text { Expensive } \\ 1 & 2 & 3 & 4 & 5 & 6 & 7 \\ \text { Light } & & & & & & \text { Heavy }\end{array}$

6. What is the weight of a humming bird?

$\begin{array}{lllllll}\text { Inexpensive } & & & & & \text { Expensive } \\ 1 & 2 & 3 & 4 & 5 & 6 & 7 \\ \text { Light } & & & & & \text { Heavy }\end{array}$

7. What is the weight of a penny?

$\begin{array}{lllllll}\text { Inexpensive } & & & & & \text { Expensive } \\ 1 & 2 & 3 & 4 & 5 & 6 & 7 \\ \text { Light } & & & & & & \text { Heavy }\end{array}$


8. What is the price of a loaf of bread?

$\begin{array}{lllllll}\text { Inexpensive } & & & & & \text { Expensive } \\ 1 & 2 & 3 & 4 & 5 & 6 & 7 \\ \text { Light } & & & & & & \text { Heavy }\end{array}$

9. What is the price of a ticket to a Broadway show?

$\begin{array}{lllllll}\text { Inexpensive } & & & & & \text { Expensive } \\ 1 & 2 & 3 & 4 & 5 & 6 & 7 \\ \text { Light } & & & & & & \text { Heavy }\end{array}$

10. What is the price of a vacation in Hawaii?

$\begin{array}{lllllll}\text { Inexpensive } & & & & & \text { Expensive } \\ 1 & 2 & 3 & 4 & 5 & 6 & 7 \\ \text { Light } & & & & & & \text { Heavy }\end{array}$

11. What is the weight of an airplane?

$\begin{array}{lllllll}\text { Inexpensive } & & & & & \text { Expensive } \\ 1 & 2 & 3 & 4 & 5 & 6 & 7 \\ \text { Light } & & & & & & \text { Heavy }\end{array}$

12. What is the weight of a pen?

$\begin{array}{lllllll}\text { Inexpensive } & & & & & \text { Expensive } \\ 1 & 2 & 3 & 4 & 5 & 6 & 7 \\ \text { Light } & & & & & & \text { Heavy }\end{array}$

13. What is the price of university textbooks?

$\begin{array}{lllllll}\text { Inexpensive } & & & & & \text { Expensive } \\ 1 & 2 & 3 & 4 & 5 & 6 & 7 \\ \text { Light } & & & & & & \text { Heavy }\end{array}$

14. What is the weight of an orange?

$\begin{array}{lllllll}\text { Inexpensive } & & & & & \text { Expensive } \\ 1 & 2 & 3 & 4 & 5 & 6 & 7 \\ \text { Light } & & & & & & \text { Heavy }\end{array}$

15. What is the weight of a black bear?

$\begin{array}{lllllll}\text { Inexpensive } & & & & & \text { Expensive } \\ 1 & 2 & 3 & 4 & 5 & 6 & 7 \\ \text { Light } & & & & & & \text { Heavy }\end{array}$

16. What is the average price of cellular phone?

Inexpensive

$\begin{array}{llll}1 & 2 & 3 & 4\end{array}$




\section{Bibliography}

Anderson, J. \& Bower, G.H. (1973). Human Associative Memory. Washington, DC: Winston.

Bargh, J.A., \& Chartrand, T.L. (2000). The mind in the middle: A practical guide to priming and automaticity research. In H.T. Reis \& C.M. Judd (Eds.), Handbook of research methods in social and personality psychology (pp. 253285). New York: Cambridge University Press.

Bower, G. H. (1981). Mood and memory. American Psychologist, 36, 129-148.

Brewer, N. T., \& Chapman, G. B. (2002). The fragile basic anchoring effect. Journal of Behavioral Decision-making, 15, 65-77.

Cervone, D., \& Peake, P. K. (1986). Anchoring, efficacy, and action: the influence of judgmental heuristics and self-efficacy judgments and behavior. Journal of Personality and Social Psychology, 50, 492-501.

Chapman, G. B., \& Bornstein, B. H. (1996). The more you ask for, the more you get: Anchoring in personal injury verdicts. Applied Cognitive Psychology, 10, 519-540.

Chapman, G. B., \& Johnson, E. J. (1999). Anchoring, activation and the construction of values. Organizational Behaviors and Human Decision Processes, 79, $115-153$.

Chun, W. Y., Spiegel, S., \& Kruglanski, A. W. (2002). Assimilative behavior identification can also be resource-dependent: A unimodel perspective on personal-attribution phases. Journal of Personality and Social Psychology, 83, 542-555.

Englich, B., \& Mussweiler, T. (2001). Sentencing under uncertainty: Anchoring effects in the courtroom. Journal of Applied Social Psychology, 31, 15351551 .

Erb, H. P., Fishbach, A., \& Kruglanski, A. W. (2002). The effects of rule priming on social judgment. Unpublished draft, University of Jena.

Fazio, R. H. (1990). A practical guide to the use of response latency in social psychological research. In C. Hendrick \& M. S. Clark (Eds.), Research methods in personality and social psychology (pp. 74-97). Newbury Park, CA: Sage. 
Festinger, L. (1954) A theory of social comparison processes, Human Relations, 7, $117-40$.

Grice, H. P. (1975). Logic and conversation. In P. Cole \& J. L. Morgan (Eds.), Syntax and semantics 3: Speech acts (pp. 41-58). New York: Academic Press.

Herr, P. M., Sherman, S. J., \& Fazio, R. H. (1983). On the consequences of priming: Assimilation and contrast effects. Journal of Experimental Social Psychology, 19, 323-340.

Higgins, E. T. (1996). Knowledge activation: Accessibility, applicability and salience. In E. T. Higgins \& A. W. Kruglanski (Eds.), Social psychology: Handbook of basic principles (pp. 133-168). New York: Guilford Press.

Higgins, E. T., Rholes, W. S., \& Jones, C. R. (1977). Category accessibility and impression formation. Journal of Experimental Social Psychology, 13, 141154.

Holyoak, K.J., Koh, K, \& Nisbett, R.E. (1989). A theory of condition: Inductive learning within rule-based default hierarchies. Psychological Review, 96, 315340 .

Jacowitz, K. E., \& Kahneman, D. (1995). Measures of anchoring in estimation tasks. Personality and Social Psychology Bulletin, 21, 1161-1166.

Klayman, J., \& Ha, Y. W. (1987). Confirmation, disconfirmation, and information in hypothesis testing. Psychological Review, 94, 211-228.

Martin, L. L., \& Achee, J. W. (1992). Beyond accessibility: The role of processing objectives in judgment. In L. L. Martin \& A. Tesser (Eds.), The construction of social judgments (pp. 195-216). Hillsdale, NJ: Lawrence Erlbaum Associates, Inc.

Meyer, D. E., \& Schvaneveldt, R. W. (1971). Facilitation in recognizing pairs of words: Evidence of a dependence between retrieval operations. Journal of Experimental Psychology, 90, 227-234.

Mussweiler, T., \& Strack, F. (1999a). Comparing is believing: A selective accessibility model of judgmental anchoring. In W. Stroebe \& M. Hewstone (Eds.), European review of social psychology (Vol. 10). Chichester, England: Wiley.

Mussweiler, T., \& Strack, F. (1999b). Hypothesis-consistent testing and semantic priming in the anchoring paradigm: A selective accessibility model. Journal of Experimental Social Psychology, 35, 136-164. 
Mussweiler, T., \& Strack, F. (2000a). The use of category and exemplar knowledge in the solution of anchoring tasks. Journal of Personality and Social Psychology, 78, 1038-1052.

Mussweiler, T., \& Strack, F. (2000b). Numeric judgments under uncertainty: The role of knowledge in anchoring. Journal of Experimental Social Psychology, 36, 495-518.

Mussweiler, T., Strack, F., \& Pfeiffer, T. (2000). Overcoming the inevitable anchoring effect: Considering the opposite compensates for selective accessibility. Personality and Social Psychology Bulletin, 26, 1142-1150.

Mussweiler, T., \& Strack, F. (2001a). Considering the impossible: Explaining the effects of implausible anchors. Social Cognition, 19, 145-160.

Mussweiler, T., \& Strack, F. (2001b). The semantics of anchoring. Organizational Behavior and Human Decision Processes, 86, 234-255.

Mussweiler, T. (2001). The durability of anchoring effects. European Journal of Social Psychology, 31, 431-442.

Mussweiler, T. (2002). The malleability of anchoring effects. Experimental Psychology, 49, 67-72.

Mussweiler, T. (2003). Comparison processes in social judgment: Mechanisms and Consequences. Psychological Review, 110, 472-489.

Neely, J. H. (1991). Semantic priming effects in visual word recognition: A selective review of current findings and theories. In D. Besner \& G. W. Humphreys (Eds.), Basic processes in reading (pp. 264-337). Hillsdale, NJ: Erlbaum.

Northcraft, G. B., \& Neale, M. A. (1987). Expert, amateurs, and real estate: An anchoring-and-adjustment perspective on property pricing decisions. Organizational Behavior and Human Decision Processes, 39, 228-241.

Pierro, A., Mannetti, L., Kruglanski, A.W., \& Sleeth-Keppler, D. (2004). Relevance Override: On the reduced impact of "cues" under high motivation conditions of persuasion studies. Journal of Personality and Social Psychology, 86, 251-264.

Plous, S. (1989). Thinking the unthinkable: The effects of anchoring on likelihood estimates of nuclear war. Journal of Applied Social Psychology, 19, 67-91.

Pohl, R. F., \& Hell, W. (1996). No reduction of hindsight bias after complete information and repeated testing. Organizational Behavior and Human Decision Processes, 67, 49-58. 
Quattrone, G. A. (1982). Overattribution and unit formation: When behavior engulfs the person. Journal of Personality and Social Psychology, 42, 593-607.

Quattrone, G. A., Lawrence, C. P., Warren, D. L., Souza-Silva, K., Finkel, S. E., \& Andrus, D. E. (1984). Explorations in anchoring: The effects of prior range, anchor extremity, and suggestive hints. Unpublished manuscript, Stanford University.

Sherif, M., \& Hovland, C. I. (1961). Social judgment. New Haven: Yale University Press.

Srull, T. K., \& Wyer, R. S., Jr. (1979). The role of category accessibility in the interpretation of information about persons: Some determinants and implications. Journal of Personality and Social Psychology, 37, 1660-1672.

Srull, T. K., \& Wyer, R. S., Jr. (1980). Category accessibility and social perception: Some implications for the study of person memory and interpersonal judgments. Journal of Personality and Social Psychology, 38, 841-856.

Strack, F., \& Mussweiler,T. (1997). Explaining the enigmatic anchoring effect: Mechanisms of selective accessibility. Journal of Personality and Social Psychology, 73, 437-446.

Tetlock, P. E. (1983). Accountability and complexity of thought. Journal of Personality and Social Psychology, 45, 74-83.

Tversky, A., \& Kahneman, D. (1974). Judgment under uncertainty: Heuristics and biases. Science, 185, 1124-1130.

Wegener, D. T., \& Petty, R. E. (1995). Flexible correction processes in social judgment: The role of naive theories in correction for perceived bias. Journal of Personality and Social Psychology, 68, 36-51.

Wegener, D. T., \& Petty, R. E. (1997). The flexible correction model: The role of naive theories of bias in bias correction. In M. P. Zanna (Ed.), Advances in experimental social psychology (Vol. 29, pp. 141-208). San Diego, CA: Academic.

Wegener, D. T., Petty, R. E., \& Detweiler-Bedell, B. T. (2001). Implications of attitude-change theories for numerical anchoring: Anchor plausibility and the limits of anchor effectiveness. Journal of Experimental Social Psychology, 37, 62-69.

Wilson, T. D., Houston, C., Etling, K. M., \& Brekke, N. (1996). A new look at anchoring effects: Basic anchoring and its antecedents. Journal of Experimental Psychology: General, 4, 387-402. 
Wong, K. F. E., \& Kwong, J. Y. Y. (2000). Is $7300 \mathrm{~m}$ equal to $7.3 \mathrm{~km}$ ? Same semantics but different anchoring effects. Organizational Behavior and Human Decision Processes, 82, 314-333. 